Supplement of

\title{
Global anthropogenic emissions of particulate matter including black carbon
}

5 Zbigniew Klimont ${ }^{1}$ et al.

${ }^{1}$ International Institute for Applied Systems Analysis (IIASA), Laxenburg, 2361, Austria

Correspondence to: Zbigniew Klimont (klimont@iiasa.ac.at) 


\section{S1 Comparison of temporal distribution patterns}

Fig S1.1 shows a comparison of the temporal patterns (it is an aggregate as the actual patterns are grid specific) for residential combustion sector, applied in the ECLIPSE project, with other data for selected countries.
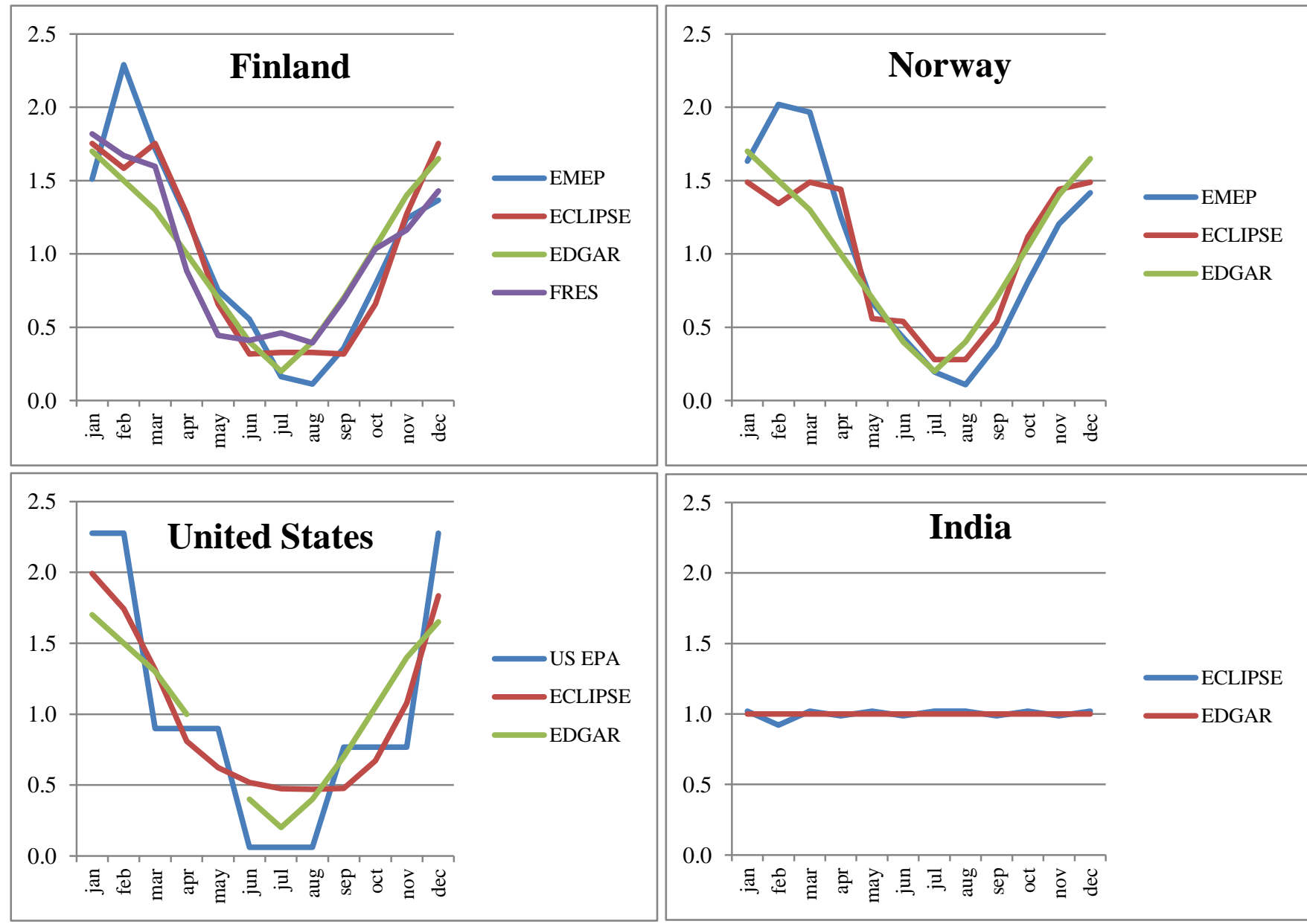

Figure S1.1. Comparison of monthly distribution of emissions used in ECLIPSE with profiles from EDGAR (EC-JRC/PBL, 2010), EMEP 5 (http://emep.int/mscw/), national Finish model FRES (Karvosenoja, 2008), and US EPA. 


\section{S2 Particulate matter emission factors for residential combustion}

The GAINS model distinguishes three principal solid fuel stove categories: traditional, improved and new stoves. Traditional heating stoves using wood or coal as fuel have simple grate based firebox designs with usually only primary air supply and no heat storing components. Consequently there is restricted availability of air for combustion and poor mixing

5 of air and pyrolysis gases. Traditional stoves in general have very high PM emission factors compared with more advanced technologies, but within this category the variability in the emission factors is also large. For example highest emission factors for traditional wood stoves have been measured in situations with restricted combustion air supply that leads to lower burn rate (Jordan and Seen, 2005). Such conditions might prevail when the user wants a lower heat supply to the room. Improved stoves have secondary air supply and heat storing components in the firebox construction that improve the

10 combustion performance and reduce emissions of PM compared with the traditional stoves. New stoves represent the most advanced stove models on the market that have firebox, construction and airflow characteristics that optimize combustion efficiency. Additionally, an electrostatic precipitator (ESP) can be fitted into the latest stoves, which further improve the PM emission performance. GAINS distinguishes also wood pellet stoves. Pellets are a very homogenous fuel and combustion is more optimized than batch fired wood log stoves and thus also the PM emissions are lower than with wood log stoves.

15 A stove heats the surrounding room, but a boiler heats water to be circulated through a piping system to heat an entire house (Johansson et al., 2004). In old-type wood log boilers up-draught combustion is commonly used, which resembles the combustion in a stove; modern wood boilers, however, use downdraught combustion and often have an isolated burn-out zone (Johansson et al., 2004). In contrast to stoves, wood boilers can be connected to a water tank to store heat, which allows the boiler to be run at a regular heat output and to certain extent optimizing the combustion conditions. Storage tanks are

20 common in modern wood boilers and also old boilers may be equipped with them, leading to lower emissions and higher efficiencies (Johansson et al., 2004). The single family house boilers are typically smaller than $50 \mathrm{~kW}_{\mathrm{th}}$, the larger residential boilers are allocated to a category medium size boilers where manual and automatic boilers are distinguished (Klimont et al., 2002; Kupiainen and Klimont, 2004, 2007). Such boilers might be an important emission source, especially when many of them are fired with coal, but there are not a of lot measurements available. The GAINS model relies on studies discussed

25 previously (EEA, 2013; Klimont et al., 2002; Kupiainen and Klimont, 2004, 2007) but for a number of countries in Europe updates were made drawing on national information provided within EU consultations (Amann et al., 2015) and recent measurements in China where 100,000s of such installations are used in both residential as well as industrial sector (Wang et al., 2009).

GAINS distinguishes also open fireplaces as a separate category which is of relevance mostly in North America and some

30 European countries, even though in Europe less than 5\% of fuelwood would be used in such installations (Klimont et al., 2002; Kupiainen and Klimont, 2004, 2007). 
Here we summarize the published measurements of emission factors for cooking and heating stoves boilers and compare them to the current ranges of region- and technology-specific GAINS values. The focus is on studies that appeared after the original development of the GAINS particulate matter module (Klimont et al., 2002; Kupiainen and Klimont, 2004, 2007).

5 Table S2.1: Summary of PM emission factors for residential wood boilers.

\begin{tabular}{|c|c|c|c|c|c|c|c|}
\hline \multicolumn{3}{|c|}{ Emission factors (mg/MJ) } & \multicolumn{4}{|c|}{ Shares $(\%)$} & \multirow[t]{2}{*}{ References } \\
\hline PM & $\mathrm{TC}^{\mathrm{a}}$ & $\mathrm{BC}$ & $\mathrm{OC}$ & $\mathrm{TC}^{\mathrm{a}}$ & $\mathrm{BC}$ & $\mathrm{OC}$ & \\
\hline \multicolumn{8}{|l|}{ wood $\log$} \\
\hline 1300 & & & & & & & (Boman et al., 2008) \\
\hline$(350-2200)^{\mathrm{b}}$ & 715 & & & 55 & & & old, no accumulator, large fuel charge \\
\hline$(73-260)^{b}$ & 60 & & & 50 & & & old, no accumulator, adjusted fuel charge \\
\hline $\begin{array}{c}95 \\
(87-100)^{b}\end{array}$ & & & & & & & (Boman et al., 2008) \\
\hline $\begin{array}{c}(87-100)^{\circ} \\
44\end{array}$ & 48 & & & 50 & & & $\begin{array}{l}\text { old, with accumulator } \\
\text { (Boman et al., 2008) }\end{array}$ \\
\hline$(11-450)^{b}$ & 18 & & & 42 & & & modern, with accumulator \\
\hline 37 & 27 & 12 & 16 & 75 & 32 & 43 & (Gaegauf et al., 2005), $35 \mathrm{~kW}$ apartment house \\
\hline $70-700$ & & 20 & $30-335$ & & & & GAINS $^{\mathrm{c}},>50 \mathrm{~kW}$, uncontrolled boiler \\
\hline $230-1300$ & & $75-200$ & $75-600$ & & & & GAINS $^{\mathrm{c}},<50 \mathrm{~kW}$, old uncontrolled boiler \\
\hline $80-520$ & & $32-50$ & $22-230$ & & & & GAINS $^{\mathrm{c}},<50 \mathrm{~kW}$, improved \\
\hline $40-260$ & & $13-37$ & $12-100$ & & & & GAINS $^{\mathrm{c}},<50 \mathrm{~kW}$, new/modern \\
\hline
\end{tabular}

wood chip

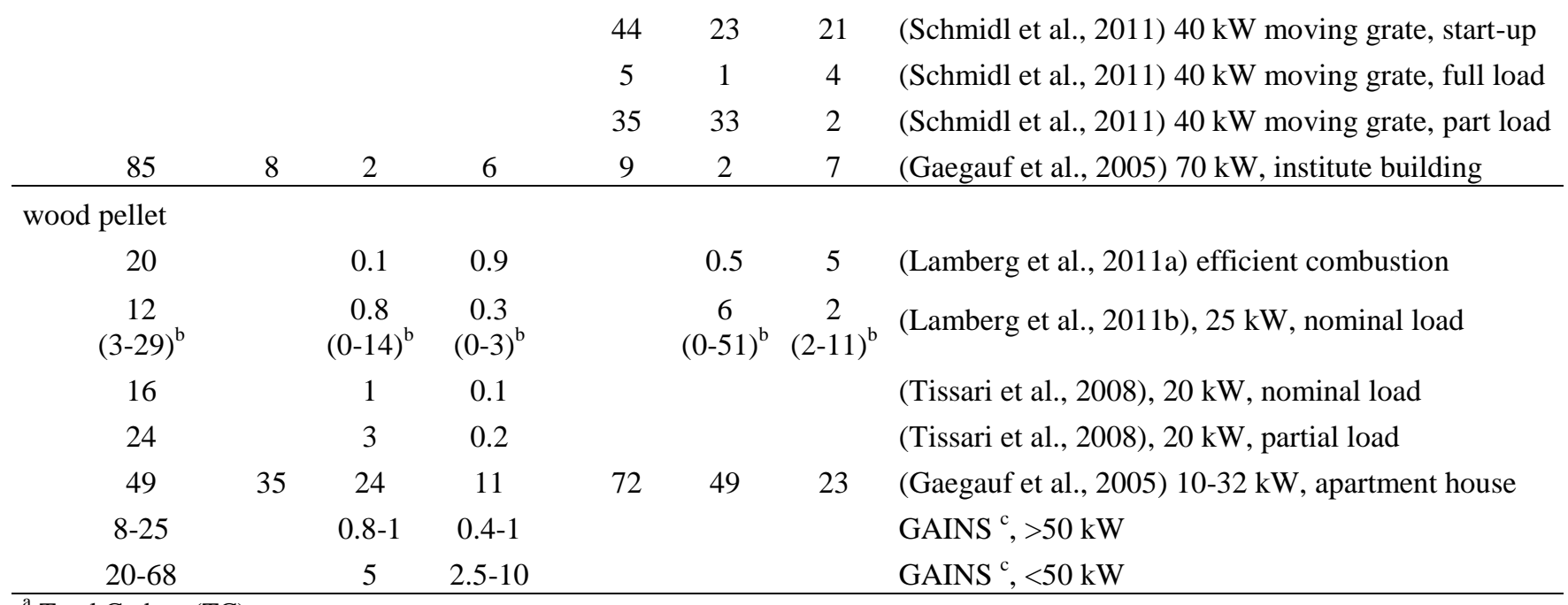

\footnotetext{
${ }^{\mathrm{a}}$ Total Carbon (TC)

b (min-max)

${ }^{\mathrm{c}} \mathrm{PM}$ value refers to PM2.5
} 
Table S2.2: Summary of PM emission factors for residential heating wood stoves.

\begin{tabular}{ccccll}
\hline \multicolumn{2}{c}{ Emission factors $(\mathrm{mg} / \mathrm{MJ})$} & \multicolumn{3}{c}{ Shares $(\%)$} & \multicolumn{2}{l}{ Reference } \\
PM & BC & OC & BC & OC & \\
\hline traditional & & & & & \\
$673-1373$ & $24-72$ & $263-623$ & $2-7$ & $39-53$ & (Alves et al., 2011) \\
$300-1400$ & - & - & $2-9$ & $35-50$ & (Gonçalves et al., 2011) incl. cold start \\
$90-900$ & - & - & $2-9$ & $35-48$ & (Gonçalves et al., 2011) incl. hot start \\
$750-1060$ & - & - & - & - & (Jordan and Seen, 2005), full airflow \\
$1560-1700$ & - & - & - & - & (Jordan and Seen, 2005), half airflow \\
$1870-3000$ & - & - & - & - & (Jordan and Seen, 2005), closed airflow \\
$128-400$ & 20 & 157 & 8 & 64 & (McDonald et al., 2000) \\
- & $39-43$ & $70-390$ & $5-14$ & $47-67$ & Studies in Kupiainen\& Klimont (2007) \\
$150^{\mathrm{a}}-930(2400)^{\mathrm{b}}$ & $32^{\mathrm{a}}-100$ & $60^{\mathrm{a}}-435(1200)^{\mathrm{b}}$ & $4-22$ & $41-50$ & GAINS; the PM value represents PM2.5
\end{tabular}
improved

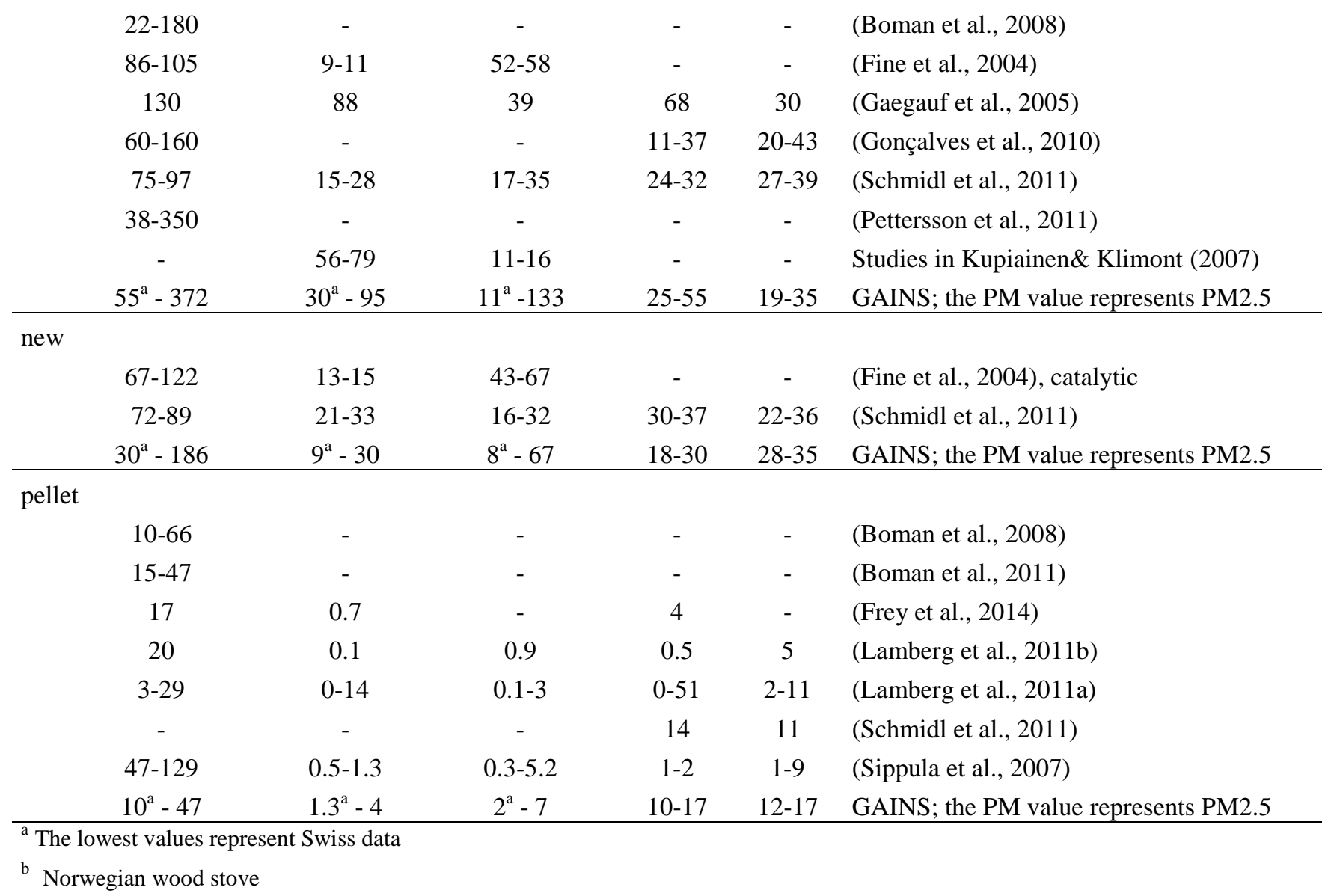


Table S2.3: Summary of PM emission factors for cookstoves using biofuels.

\begin{tabular}{|c|c|c|c|}
\hline \multicolumn{3}{|c|}{ Emission factors (mg/MJ) } & \multirow[t]{2}{*}{ References } \\
\hline $\mathrm{PM}$ & $\mathrm{BC}$ & $\mathrm{OC}$ & \\
\hline \multicolumn{4}{|l|}{ traditional } \\
\hline 530 & 44 & 250 & (Just et al., 2013) \\
\hline 106 & 50 & 44 & (Roden et al., 2009), 3-stone, lab measurements \\
\hline $\begin{array}{c}515 \\
(300-1000)^{\mathrm{a}}\end{array}$ & $\begin{array}{c}83 \\
(10-210)^{\mathrm{a}}\end{array}$ & $\begin{array}{c}254 \\
(90-660)^{\mathrm{a}}\end{array}$ & (Roden et al., 2009), Honduras, field measurements \\
\hline $\begin{array}{c}510 \\
(280-510)^{b}\end{array}$ & $\begin{array}{c}65-75 \\
(40-75)^{\mathrm{b}}\end{array}$ & $\begin{array}{c}229 \\
(125-229)^{b}\end{array}$ & GAINS $^{c}$ \\
\hline \multicolumn{4}{|l|}{ improved } \\
\hline 150 & 80 & 20 & (Just et al., 2013), rocket stove \\
\hline $270(100-500)^{\mathrm{a}}$ & & & (Li et al., 2009), improved stoves, PM2.5 \\
\hline $\begin{array}{c}394 \\
(120-700)^{\mathrm{a}}\end{array}$ & $\begin{array}{c}102 \\
(6-325)^{a}\end{array}$ & $\begin{array}{c}208 \\
(60-460)^{\mathrm{a}}\end{array}$ & (Roden et al., 2009), improved no chimney, field measurements \\
\hline $\begin{array}{c}205 \\
(105-270)^{b}\end{array}$ & $\begin{array}{c}50-75 \\
(27-75)^{\mathrm{b}} \\
\end{array}$ & $\begin{array}{c}63 \\
(31-68)\end{array}$ & GAINS $^{c}$ \\
\hline \multicolumn{4}{|l|}{ new } \\
\hline $\begin{array}{c}255 \\
(40-720)^{\mathrm{a}}\end{array}$ & $\begin{array}{c}116 \\
(6-660)^{a}\end{array}$ & $\begin{array}{c}93 \\
(33-370)^{\mathrm{a}}\end{array}$ & (Roden et al., 2009), improved with chimney, field measurements \\
\hline $56-102$ & $11-21$ & $19-34$ & GAINS $^{c}$ \\
\hline \multicolumn{4}{|l|}{ fan assisted } \\
\hline $\begin{array}{c}86 \\
(25-125)^{\mathrm{a}}\end{array}$ & $\begin{array}{c}33 \\
(6-100)^{a}\end{array}$ & $\begin{array}{c}38 \\
(4-71)^{\mathrm{a}}\end{array}$ & (Roden et al., 2009), fan assisted, lab measurements \\
\hline 54 & 33 & 14 & (Just et al., 2013), gasifier with fan \\
\hline 17 & 4 & 9 & GAINS $^{\mathrm{c}}$ \\
\hline
\end{tabular}

a (min-max)

${ }^{\mathrm{b}}$ central value for fuelwood and in brackets the whole range including also dung and agricultural residues

${ }^{c}$ the PM value represents PM2.5 
Table S2.4: Summary of PM emission factors for coal cooking and heating stoves

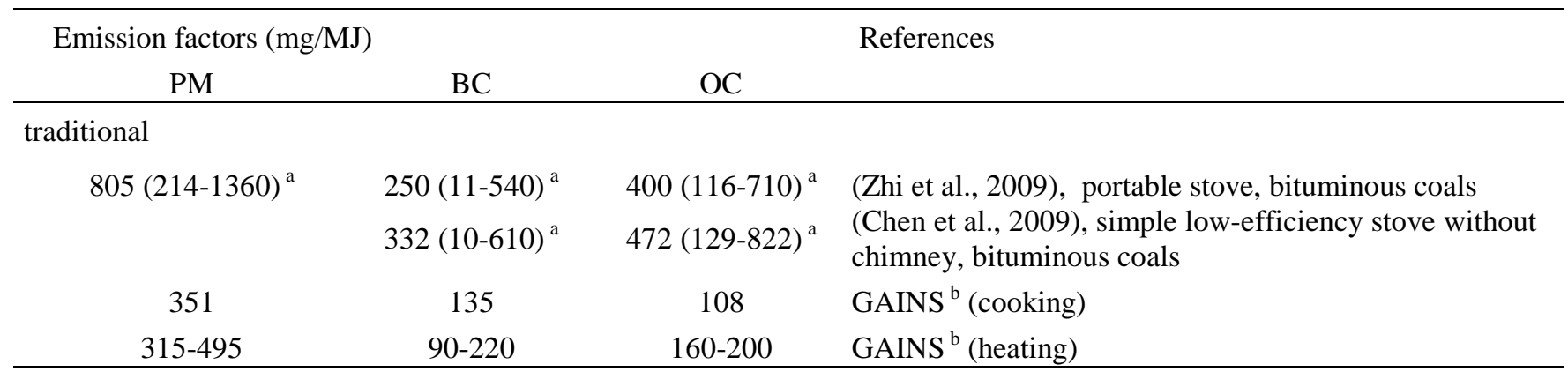

improved

\begin{tabular}{|c|c|c|c|}
\hline & $466(6-1377)^{a}$ & $248(35-551)^{a}$ & (Chen et al., 2009), high-efficiency stove with chimney \\
\hline 492 & 183 & 200 & (Zhang et al., 2008), steel stove, brown coal \\
\hline 36 & 1 & 16 & (Zhang et al., 2008), steel stove, bituminous coal \\
\hline 246 & 132 & 60 & GAINS $^{\text {b }}$ (cooking) \\
\hline $315-350$ & $82-200$ & $88-112$ & GAINS $^{\mathrm{b}}$ (heating) \\
\hline 270 & 23 & 96 & (Li et al., 2016), average for bituminous coals \\
\hline 176 & 108 & 32 & GAINS $^{\mathrm{b}}$ (cooking) \\
\hline $158-248$ & $73-176$ & $48-60$ & GAINS $^{\mathrm{b}}$ (heating) \\
\hline \multicolumn{4}{|l|}{ briquettes } \\
\hline $440(98-930)^{\mathrm{a}}$ & $12(2-23)^{a}$ & $233(67-460)^{a}$ & (Zhi et al., 2009), traditional portable stove \\
\hline $202(90-346)^{\mathrm{a}}$ & $2(0.5-6)^{\mathrm{a}}$ & $124(36-217)^{\mathrm{a}}$ & (Zhi et al., 2009), improved stove with chimney \\
\hline 17 & 0.4 & 6.5 & (Li et al., 2016), semi-coke briquettes \\
\hline $23-135$ & $0.3-1$ & $9-55$ & GAINS $^{b}$ \\
\hline
\end{tabular}

\footnotetext{
a (min-max)

${ }^{\mathrm{b}}$ the PM value represents PM2.5
} 
Table S3.1: Summary of PM emission factors for diesel generator sets

\begin{tabular}{|c|c|c|c|c|c|}
\hline \multicolumn{3}{|c|}{ Emission factors (mg/MJ) } & \multicolumn{2}{|c|}{ Shares $(\%)$} & \multirow[t]{2}{*}{ Reference } \\
\hline $\mathrm{PM}$ & $\mathrm{BC}$ & $\mathrm{OC}$ & $\mathrm{BC}$ & $\mathrm{OC}$ & \\
\hline $69-189$ & & & & & Uma et al. (2004), $10 \mathrm{~kW}$ (higher value), $40 \mathrm{~kW}$ (lower value) \\
\hline 139 & & & $66 \%$ & & Bond et al. (2004) \\
\hline \multirow[t]{2}{*}{$13 / 22$} & & & & & Gilmore et al. (2006), ICE $10 \mathrm{~kW}$, with/without DPF \\
\hline & & $116-585$ & & & Watson et al. (2006) ${ }^{\mathrm{a}}$ \\
\hline $59-190$ & $12-54$ & $30-120$ & $31 \%$ & $51 \%$ & Shah et al. (2007) ${ }^{\mathrm{b}} 300 \mathrm{~kW} 1985$ Detroit Diesel V92, 2-str \\
\hline 45-219 & $30-145$ & $8-56$ & $67 \%$ & $21 \%$ & Shah et al. (2007) ${ }^{\mathrm{b}} 350 \mathrm{~kW} 2000$ Cat 3406C, 4-str \\
\hline $22-143$ & $10-80$ & $6-37$ & $53 \%$ & $25 \%$ & Shah et al. (2007) ${ }^{\mathrm{b}} 300 \mathrm{~kW} 1985$ Detroit Diesel V92, 2-str, DOC \\
\hline $59-203$ & $28-145$ & $4-16$ & $67 \%$ & $8 \%$ & Shah et al. (2007) ${ }^{\mathrm{b}} 350 \mathrm{~kW} 2000$ Cat 3406C, 4-str, DOC \\
\hline $23-190$ & $9-96$ & $10-81$ & $49 \%$ & $36 \%$ & $\begin{array}{l}\text { Shah et al. (2007) }{ }^{\mathrm{b}} 300 \mathrm{~kW} 1985 \text { Detroit Diesel V92, 2-str, } \\
\text { DOC+FBC }\end{array}$ \\
\hline $4-26$ & $2.5-19$ & $1-3$ & $76 \%$ & $15 \%$ & Shah et al. (2007) ${ }^{\mathrm{b}} 350 \mathrm{~kW} 2000$ Cat 3406C, 4-str, passive-DPF \\
\hline \multirow[t]{2}{*}{$1-3$} & $0.8-2$ & $1-6$ & $67 \%$ & $49 \%$ & Shah et al. (2007) ${ }^{\mathrm{b}} 350 \mathrm{~kW} 2000$ Cat $3406 \mathrm{C}$, 4-str, active-DPF \\
\hline & & & & $20-70 \%$ & Watson et al. (2008) \\
\hline $14-42$ & & & & & Zhu et al. (2009) ${ }^{\mathrm{c}}$ \\
\hline $174-433$ & & & & & Tsai et al. (2010) ${ }^{\mathrm{d}}$ \\
\hline 55 & & & & & Anayochukwu et al. (2013) \\
\hline
\end{tabular}

GAINS emission factors; the PM value represents PM2.5

$\begin{array}{cccccl}96 & 40 & 28 & 41 \% & 29 \% & \text { No control } \\ 48-64 & 20-26 & 14-19 & 41 \% & 29 \% & \text { Controlled, no DPF } \\ <1-3 & 0.5-2 & 0.3-0.8 & & & \text { Controlled, with DPF }\end{array}$

${ }^{2}$ Higher value with $10 \%$ load and lower value with $100 \%$ load for a $100 \mathrm{~kW}$ DG set

5 bower value with $100 \%$ load and higher value with $10 \%$ load, share of $\mathrm{BC} / \mathrm{OC}$ is average of all loads

${ }^{c}$ Average of 14 military diesel generators with rated capacities of $10,30,60$, and $100 \mathrm{~kW}$ under different load conditions. The fleet average EFs are $1.2+/-0.6 \mathrm{~g} / \mathrm{kg}$ for PM.

${ }^{\mathrm{d}}$ Higher value with no load and lower value with $10 \mathrm{~kW}$ 


\section{S4 Transport sector}

Table S4.1: Comparison of selected measured emissions factors and ranges used in the GAINS model for diesel and gasoline cars and 5 light duty vehicles.

\begin{tabular}{|c|c|c|c|c|}
\hline & \multicolumn{3}{|c|}{ Emission factors (mg/MJ) } & \multirow[t]{2}{*}{ Reference } \\
\hline & $\mathrm{PM}$ & $\mathrm{BC}$ & $\mathrm{OC}$ & \\
\hline \multicolumn{5}{|l|}{ Diesel } \\
\hline Pre-/early regulation & $44-67$ & $9-17$ & $13-34$ & (Subramanian et al., 2009) \\
\hline Euro 1 & 67 & 17 & 13 & (Subramanian et al., 2009) \\
\hline Euro 2 & $30-33$ & $7-16$ & $8-12$ & (Cheung et al., 2009; Subramanian et al., 2009) \\
\hline Euro 3 & $10-29$ & & & (Graham, 2005) \\
\hline Euro 4 & $6-11$ & $3-8$ & $1-2$ & (Cheung et al., 2009; Geller et al., 2006) \\
\hline Euro 4 with DPF & $0.2-0.3$ & $0.02-0.1$ & $0.02-0.06$ & (Dwyer et al., 2010; Louis et al., 2016) \\
\hline Pre-/early regulation & $56-133$ & $38-76$ & $21-51$ & GAINS $^{a}$ \\
\hline Euro 1 & $22-50$ & $16-35$ & $5-11$ & $\begin{array}{l}\text { GAINS }{ }^{\text {a }} \text {; for developing countries the values only } \\
\text { marginally lower than pre/early regulation }\end{array}$ \\
\hline Euro 2 & $15-40$ & $12-32$ & $3-6$ & $\begin{array}{l}\text { GAINS }{ }^{\text {a }} \text {; for developing countries the values only } \\
\text { marginally lower than pre/early regulation }\end{array}$ \\
\hline Euro 3 & $11-29$ & $10-22$ & $1-2$ & GAINS $^{\text {a }}$ \\
\hline Euro 4 & $5-20$ & $4-17$ & $0.5-1.6$ & GAINS $^{a}$ \\
\hline Euro 4 with DPF & $0.5-1$ & $0.1-0.3$ & $0.1-0.6$ & GAINS $^{\text {a }}$ \\
\hline
\end{tabular}

\section{Gasoline}

\begin{tabular}{lcccl} 
Pre-/early regulation & $4-10$ & $0.5-2$ & $2-10$ & see studies in Kupiainen and Klimont $(2004,2007)$ \\
Euro 1, & $1-4$ & $0.6-1.5$ & $0.3-1.6$ & see studies in Kupiainen and Klimont $(2004,2007)$ \\
Euro 3 & $0.2-2$ & $0.01-0.2$ & $0.2-0.6$ & (Cheung et al., 2009; Geller et al., 2006; Graham, 2005) \\
Euro 4 & & $0.001-0.4$ & & (Louis et al., 2016) \\
\hline Pre-/early regulation & 6 & 1 & $3-4$ & GAINS $^{\text {a }}$ \\
Euro 1, 2 & $1-4$ & $0.2-1$ & $0.3-1.7$ & GAINS $^{\text {a }}$ \\
Euro 3, 4 & $0.3-1.1$ & $0.05-0.5$ & $0.1-0.4$ & GAINS $^{\text {a }}$ \\
\hline
\end{tabular}

${ }^{\mathrm{a}}$ the PM value represents PM2.5 
Table S4.2: Comparison of selected measured emissions factors and ranges used in the GAINS model for diesel heavy duty vehicles

\begin{tabular}{|c|c|c|c|c|}
\hline & \multicolumn{3}{|c|}{ Emission factors (mg/MJ) } & \multirow[t]{2}{*}{ Reference } \\
\hline & $\mathrm{PM}$ & $\mathrm{BC}$ & $\mathrm{OC}$ & \\
\hline \multicolumn{5}{|l|}{ Diesel heavy duty trucks } \\
\hline \multirow[t]{3}{*}{ Pre-/early regulation } & $28-33$ & & & (Herner et al., 2009; Yanowitz et al., 2000) \\
\hline & $44-244$ & $4-50$ & $15-122$ & (Subramanian et al., 2009), Bangkok, Thailand \\
\hline & $30-50$ & & & (Liu et al., 2009), on-road measurements in China \\
\hline \multirow[t]{3}{*}{ Euro I } & 11 & & & (Yanowitz et al., 2000) \\
\hline & 22 & 4 & 9 & (Subramanian et al., 2009), Bangkok, Thailand \\
\hline & $10-20$ & & & (Liu et al., 2009), on-road measurements in China \\
\hline \multirow[t]{2}{*}{ Euro II } & $22-44$ & $2-9$ & $7-22$ & (Subramanian et al., 2009), Bangkok, Thailand \\
\hline & $7-17$ & 16 & & $\begin{array}{l}\text { measurements in China } \\
\text { (Liu et al., 2009; Wang et al., 2011), on-road }\end{array}$ \\
\hline Euro III & $3-7$ & 9 & & measurements in China \\
\hline Euro IV & & 4 & & (Wang et al., 2011), on-road measurements in China \\
\hline Pre-/early regulation & $34-107$ & $17-53$ & $10-37$ & GAINS $^{\text {a }}$ \\
\hline Euro I & $21-71$ & $17-53$ & $6-19$ & GAINS $^{\text {a }}$ \\
\hline Euro II & $11-44$ & $7-30$ & $2-10$ & GAINS $^{a}$ \\
\hline Euro III & $10-27$ & $8-25$ & $2-7$ & GAINS $^{\text {a }}$ \\
\hline Euro IV, V & $2-7$ & $2-5$ & $0.3-1$ & GAINS $^{\text {a }}$ \\
\hline Euro VI & $0.1-0.4$ & $0.01-0.06$ & $0.06-0.15$ & GAINS $^{\mathrm{a}}$ \\
\hline
\end{tabular}

${ }^{\mathrm{a}}$ the PM value represents PM2.5 
Table S4.3: Comparison of selected measured emissions factors and ranges used in the GAINS model for non-road machinery.

\begin{tabular}{|c|c|c|c|c|}
\hline & \multicolumn{3}{|c|}{ Emission factors (mg/MJ) } & \multirow[t]{2}{*}{ Reference } \\
\hline & PM & $\mathrm{BC}$ & $\mathrm{OC}$ & \\
\hline \multicolumn{5}{|l|}{ Diesel locomotives } \\
\hline Pre-/early regulation & $49-67$ & & & $\begin{array}{l}\text { (Dincer and Elbir, 2007; Johnson et al., 2013; Tang et } \\
\text { al., 2015) }\end{array}$ \\
\hline \multirow[t]{4}{*}{ Regulated } & $20-40$ & 20 & & $\begin{array}{l}\text { (Dincer and Elbir, 2007; Galvis et al., 2013; Johnson et } \\
\text { al., 2013; Tang et al., 2015) }\end{array}$ \\
\hline & 30 & 14 & & (Galvis et al., 2013) \\
\hline & 20 & 15 & & (Jaffe et al., 2014) \\
\hline & 37 & 21 & & (Krasowsky et al., 2015) \\
\hline pre-regulated & $49-98$ & $24-45$ & $12-25$ & GAINS $^{\mathrm{a}}$ \\
\hline regulated (stage I) & $26-49$ & $11-22$ & $6-12$ & GAINS $^{\text {a }}$ \\
\hline \multicolumn{5}{|l|}{ Agriculture } \\
\hline \multirow[t]{2}{*}{ Pre-regulation } & 141 & 58 & 41 & (Kupiainen and Klimont, 2007) \\
\hline & 89 & 49 & & (EEA, 2013) \\
\hline Stage I & $20-39$ & $16-21$ & & (EEA, 2013) \\
\hline Stage II & 15 & 11.5 & & (EEA, 2013) \\
\hline Pre-regulation & $100-170$ & $41-70$ & $29-50$ & GAINS $^{\text {a }}$ \\
\hline Stage I & $57-96$ & $23-40$ & $16-27$ & GAINS $^{\mathrm{a}}$ \\
\hline Stage II, III & $27-43$ & $10-19$ & $8-12$ & GAINS $^{\mathrm{a}}$ \\
\hline Stage IV,V & $6-10$ & $0.7-1.2$ & $0.5-0.8$ & GAINS $^{\mathrm{a}}$ \\
\hline \multicolumn{5}{|l|}{ Construction } \\
\hline \multirow[t]{2}{*}{ Pre-regulation } & 140 & 65 & 30 & (Kupiainen and Klimont, 2007) \\
\hline & 103 & 56 & & (EEA, 2013) \\
\hline Stage I & 85 & 47 & & (EEA, 2013) \\
\hline Pre-regulation & $95-140$ & $46-68$ & $21-31$ & GAINS $^{\mathrm{a}}$ \\
\hline Stage I & $57-76$ & $26-39$ & $12-18$ & GAINS $^{\mathrm{a}}$ \\
\hline Stage II, III & $24-36$ & $12-17$ & $5-8$ & GAINS $^{a}$ \\
\hline Stage IV,V & $6-8$ & $0.8-1.2$ & $0.4-0.6$ & GAINS $^{\mathrm{a}}$ \\
\hline
\end{tabular}

${ }^{\mathrm{a}}$ the PM value represents PM2.5 
Table S4.4: Comparison of selected measured emissions factors and ranges used in the GAINS model for 2-wheelers.

\begin{tabular}{|c|c|c|c|c|}
\hline & \multicolumn{3}{|c|}{ Emission factors (mg/MJ) } & \multirow[t]{2}{*}{ References } \\
\hline & $\mathrm{PM}$ & $\mathrm{BC}$ & $\mathrm{OC}$ & \\
\hline \multicolumn{5}{|l|}{ 2-stroke } \\
\hline \multirow[t]{2}{*}{ Euro 0 mopeds } & $250(198-295)$ & & & (Spezzano et al., 2008), hot start \\
\hline & $160(121-878)$ & & & (Spezzano et al., 2008), cold start \\
\hline \multirow[t]{2}{*}{ Euro 1 mopeds } & $169(102-235)$ & & & (Spezzano et al., 2008), hot start \\
\hline & $42(26-71)$ & & & (Spezzano et al., 2008), cold start \\
\hline \multirow[t]{2}{*}{ Euro 2 mopeds } & $147-217$ & & & (Spezzano et al., 2008), hot start \\
\hline & $13-215$ & & & (Spezzano et al., 2008), cold start \\
\hline CNG rickshaw, Delhi, India & $124-160$ & & & (Grieshop et al., 2012) \\
\hline Euro 0 mopeds & $132-1400$ & $10-75$ & $90-1015$ & GAINS $^{a}$ \\
\hline Euro 1 mopeds & $12-450$ & $7-49$ & $40-300$ & GAINS $^{\text {a }}$ \\
\hline Euro 2 mopeds & $37-280$ & $6-45$ & $23-172$ & GAINS $^{\text {a }}$ \\
\hline Euro 3 mopeds & $14-112$ & $3-30$ & $8-61$ & GAINS $^{\text {a }}$ \\
\hline \multicolumn{5}{|l|}{ 4-stroke } \\
\hline Motorcycles & $2.6-3.7$ & & & (Yang et al., 2005), cold start \\
\hline Euro 0 motorcycles & 4 & & & (Spezzano et al., 2007) \\
\hline Euro 1 motorcycles & 2 & & & (Spezzano et al., 2007) \\
\hline Rickshaw, Delhi, India & $30-45$ & & & (Grieshop et al., 2012) \\
\hline CNG rickshaw, Delhi, India & $12-13$ & & & (Grieshop et al., 2012) \\
\hline Euro 0 motorcycles & $6-14$ & $1-2$ & $3-9$ & GAINS $^{\text {a }}$ \\
\hline Euro 1 motorcycles & $5-12$ & $1-2$ & $2-7$ & GAINS $^{\text {a }}$ \\
\hline Euro 2 motorcycles & $3-5$ & $0.5-0.8$ & $0.4-1.7$ & GAINS $^{\text {a }}$ \\
\hline Euro 3 motorcycles & $2-3$ & $0.5-0.75$ & $0.3-1.4$ & GAINS $^{\text {a }}$ \\
\hline
\end{tabular}

${ }^{\mathrm{a}}$ the PM value represents PM2.5 
Table S4.5: Summary of PM emission factor ranges used in the GAINS model for non-exhaust transport sources

\begin{tabular}{lcccc}
\hline & $\begin{array}{c}\text { Emission factors }(\mathrm{mg} / \mathrm{km}) \\
\mathrm{PM}_{10}\end{array}$ & $\mathrm{PM}_{2.5}$ & $\mathrm{BC}$ & $\mathrm{OC}$ \\
\hline Brake wear & & & & \\
$\quad$ Cars & $3.5-12$ & $2.5-5$ & $0.05-0.12$ & $0.8-2.2$ \\
$\quad$ Light duty vehicles & $3.5-19$ & $2.5-8$ & $0.05-0.2$ & $0.8-3.5$ \\
$\quad$ Heavy duty vehicles & $21-53$ & $13-21$ & $0.25-0.5$ & $5-17$ \\
\hline Tyre wear & & & & $0.5-2.4$ \\
$\quad$ Cars & $1.5-9$ & $0.15-0.7$ & $0.2-1$ & $0.85-2.4$ \\
Light duty vehicles & $2.5-7$ & $0.2-0.7$ & $0.35-1$ & $15-17$ \\
$\quad$ Heavy duty vehicles & $40-47$ & $4.2-4.7$ & $6-7$ & $0.7-1$ \\
\hline Road abrasion & $7-10$ & & & $4-14^{\mathrm{a}}$ \\
$\quad$ Cars \& Light duty vehicles & $30-140^{\mathrm{a}}$ & $20-80^{\mathrm{a}}$ & $0.2-1.5^{\mathrm{a}}$ & $3-5$ \\
$\quad$ Heavy duty vehicles & $38-50$ & $18-27$ & $0.7-1$ & $0.15-0.6$ \\
\hline
\end{tabular}

${ }^{\mathrm{a}}$ vehicles with studded tires; variation between estimates for Scandinavian and alpine countries 


\section{S5 Industry}

GAINS model PM emission factors (as used for the ECLIPSE V5a) for brick making compared with values used in GAINS previously (UNEP/WMO, 2011) and recent set of measurements on typical kilns used in South Asia (Weyant et al., 2014).

5 Table S5.1: Comparison of emissions factors used in the GAINS model for brick kilns with selected other studies.

\begin{tabular}{|c|c|c|c|}
\hline \multicolumn{3}{|c|}{ Emission factors ( $\mathrm{g} \mathrm{kg}^{-1}$ brick) } & \multirow[t]{2}{*}{ References } \\
\hline PM2.5 & BC & OC & \\
\hline \multicolumn{4}{|l|}{ Clamp kiln } \\
\hline 1.6 & 0.35 & 0.3 & $(\mathrm{UNEP} / \mathrm{WMO}, 2011)^{\mathrm{a}}$ \\
\hline 1 & 0.3 & 0.1 & GAINS (Asia) \\
\hline 1 & 0.35 & 0.15 & GAINS (Latin America and Africa) \\
\hline \multicolumn{4}{|l|}{ Downdraft kiln } \\
\hline 0.49 & 0.19 & 0.07 & (Weyant et al., 2014) \\
\hline 0.97 & 0.29 & 0.09 & GAINS (all regions) \\
\hline \multicolumn{4}{|l|}{ Bull's trench kiln (BTK) } \\
\hline 1.31 & 0.27 & 0.24 & $(\mathrm{UNEP} / \mathrm{WMO}, 2011)^{\mathrm{a}}$ \\
\hline $0.19(0.08-0.33)$ & $0.15(0.09-0.27)$ & 0.007 & (Weyant et al., 2014) $^{\mathrm{b}}$ \\
\hline $0.18 / 0.8$ & $0.13 / 0.25$ & $0.01 / 0.07$ & GAINS (Asia); fixed /moving chimney \\
\hline \multicolumn{4}{|l|}{ Vertical shaft brick kiln (VSBK) } \\
\hline 0.77 & 0.175 & 0.15 & $(\mathrm{UNEP} / \mathrm{WMO}, 2011)^{\mathrm{a}}$ \\
\hline $0.07(0.005-0.009)$ & $0.0015(0.001-0.002)$ & 0.014 & $\left(\right.$ Weyant et al., 2014) ${ }^{\mathrm{b}}$ \\
\hline 0.093 & $0.001-0.004$ & $0.002-0.059$ & GAINS (Asia) \\
\hline 0.093 & 0.002 & 0.059 & GAINS (Latin America and Africa) \\
\hline \multicolumn{4}{|l|}{ Zig-zag kiln } \\
\hline $0.06(0.03-0.06)$ & $0.01(0.014-0.03)$ & 0.005 & $\left(\right.$ Weyant et al., 2014) ${ }^{\mathrm{b}}$ \\
\hline 0.13 & 0.04 & 0.02 & GAINS (Asia) \\
\hline \multicolumn{4}{|l|}{ Tunnel kiln (coal) } \\
\hline 0.28 & 0.0035 & 0.003 & $(\mathrm{UNEP} / \mathrm{WMO}, 2011)^{\mathrm{a}}$ \\
\hline 0.24 & 0.01 & $<0.00$ & (Weyant et al., 2014) \\
\hline 0.18 & 0.002 & 0.0035 & GAINS (all regions) \\
\hline \multicolumn{4}{|l|}{ Hoffman kiln } \\
\hline 0.08 & 0.003 & 0.005 & GAINS (all regions) \\
\hline \multicolumn{4}{|l|}{ Marquez kiln (MK) } \\
\hline 0.15 & 0.06 & 0.02 & GAINS (Latin America) \\
\hline
\end{tabular}

${ }^{a}$ Previous version of the GAINS model was used

${ }^{\mathrm{b}}$ Central value and ranges of average values; all measurement data provided in the original study 
Brick sector production structure in Asia has been analysed in a number of studies addressing either the whole region where selected countries, typically key producers including China, India, Pakistan, Bangladesh, Vietnam, are discussed (AIT, 2003; BASIN, 1999; FAO, 1993; Heierli and Maithel, 2008; Maithel, 2014) or focusing on particular countries like China (Zhang, 1997), India (BASIN, 1998; Maithel et al., 2012; Verma and Uppal, 2013), Bangladesh (Croitoru and Sarraf, 2012;

5 Guttikunda et al., 2013; World Bank, 2011), Cambodia (Rozemuller, 1999), Afghanistan (Samuel Hall Consulting, 2011), Nepal (Heierli et al., 2007). More recently, a number of development programs and local air pollution studies focused on this sector in the Latin America and Caribbean regions, including some where information about kiln structure was collected (Bellprat, 2009; EELA, 2011; Erbe, 2011; PRAL, 2012; Stratus Consulting, 2014; SwissContact, 2014a). Fewer assessments exist for Africa (Scott, 2013; SwissContact, 2014c). The updated and country specific data for Latin America and Caribbean

10 (LAC) is included only in version V5a of ECLIPSE since the previous versions included just five regions for the whole LAC; Argentina, Brazil, Chile, Mexico, other LAC.

GAINS activity data has been built on the basis of several regional studies where production, energy efficiency, and sector structure were discussed, i.e., Asia (AIT, 2003; Co et al., 2009; Croitoru and Sarraf, 2012; FAO, 1993; Guttikunda et al., 2013; Heierli et al., 2007; Heierli and Maithel, 2008; Maithel, 2014; Maithel et al., 2012; Samuel Hall Consulting, 2011;

15 Subrahmanya, 2006; Verma and Uppal, 2013; World Bank, 2011; Zhang, 1997), Africa (Alam, 2006; Scott, 2013; SwissContact, 2014c), Latin America and Caribbean (Bellprat, 2009; EELA, 2011; PRAL, 2012; Stratus Consulting, 2014; SwissContact, 2014b). For several countries where we found no regional analysis, the United Nations data on 'building bricks, made of clay' was used (http://unstats.un.org/unsd/industry/commoditylist2.asp). There are some differences between different versions of the ECLIPSE datasets; specifically during the development of the $V 5 a$ version, the data for all countries

20 in Latin America and Caribbean was revisited and updated, and a new version of the UN statistics was downloaded.

Table S5.2: Brick production in key regions; GAINS model assumptions - ECLIPSE V5a, Tg bricks year ${ }^{-1}$

\begin{tabular}{|c|c|c|c|c|c|}
\hline & 1990 & 1995 & 2000 & 2005 & 2010 \\
\hline Global & 1542 & 2357 & 2688 & 3022 & 3574 \\
\hline $\begin{array}{l}\text { Asia } \\
\text { of which: }\end{array}$ & 1314 & 2130 & 2530 & 2819 & 3320 \\
\hline China & 1050 & 1800 & 2106 & 2204 & 2508 \\
\hline India & 131 & 178 & 254 & 406 & 553 \\
\hline Vietnam & 20 & 20 & 27 & 46 & 65 \\
\hline Bangladesh & 9 & 15 & 18 & 17 & 25 \\
\hline Pakistan & 32 & 41 & 50 & 59 & 74 \\
\hline Other Asia & 71 & 75 & 76 & 87 & 95 \\
\hline Africa & 18 & 18 & 15 & 17 & 22 \\
\hline Europe & 158 & 156 & 72 & 82 & 79 \\
\hline Latin America and Caribbean & 29 & 30 & 43 & 75 & 127 \\
\hline Other & 23 & 23 & 27 & 29 & 25 \\
\hline
\end{tabular}




\section{S6 Emissions of PM species over time in ECLIPSE datasets}

The Fig S6.1 shows emissions of PM10, PM2.5, BC, and OC calculated with the GAINS model within different versions of the ECLIPSE dataset. These have been created between 2013 and 2015 and include a number of updates to activity data and emission factors; the methodology remained the same. The changes for PM10 and PM2.5 are similar, driven by updates of

5 activity data, i.e., the energy statistics from IEA were reimported for the whole time series for the version V5 and V5a and for China the regional coal statistics were used. Control strategies have been updated continuously considering more up to date information available over time. Additionally, in version V5a Latin America and Caribbean were revised since higher spatial resolution was introduced in the GAINS model. Several of the above mentioned updates affected also emissions of $\mathrm{BC}$ and $\mathrm{OC}$ but the largest impact on the $\mathrm{BC}$ emissions was due to introduction of emissions from kerosene lamps which

10 were not specifically distinguished in $V 4 a$; this represents the key component of the higher emissions in $V 5$, V5a. For OC the change is in the opposite direction and $V 5 a$ has significantly lower emissions than previous versions which is due to update of the OC emission factor for residential cooking in Asia and Africa.
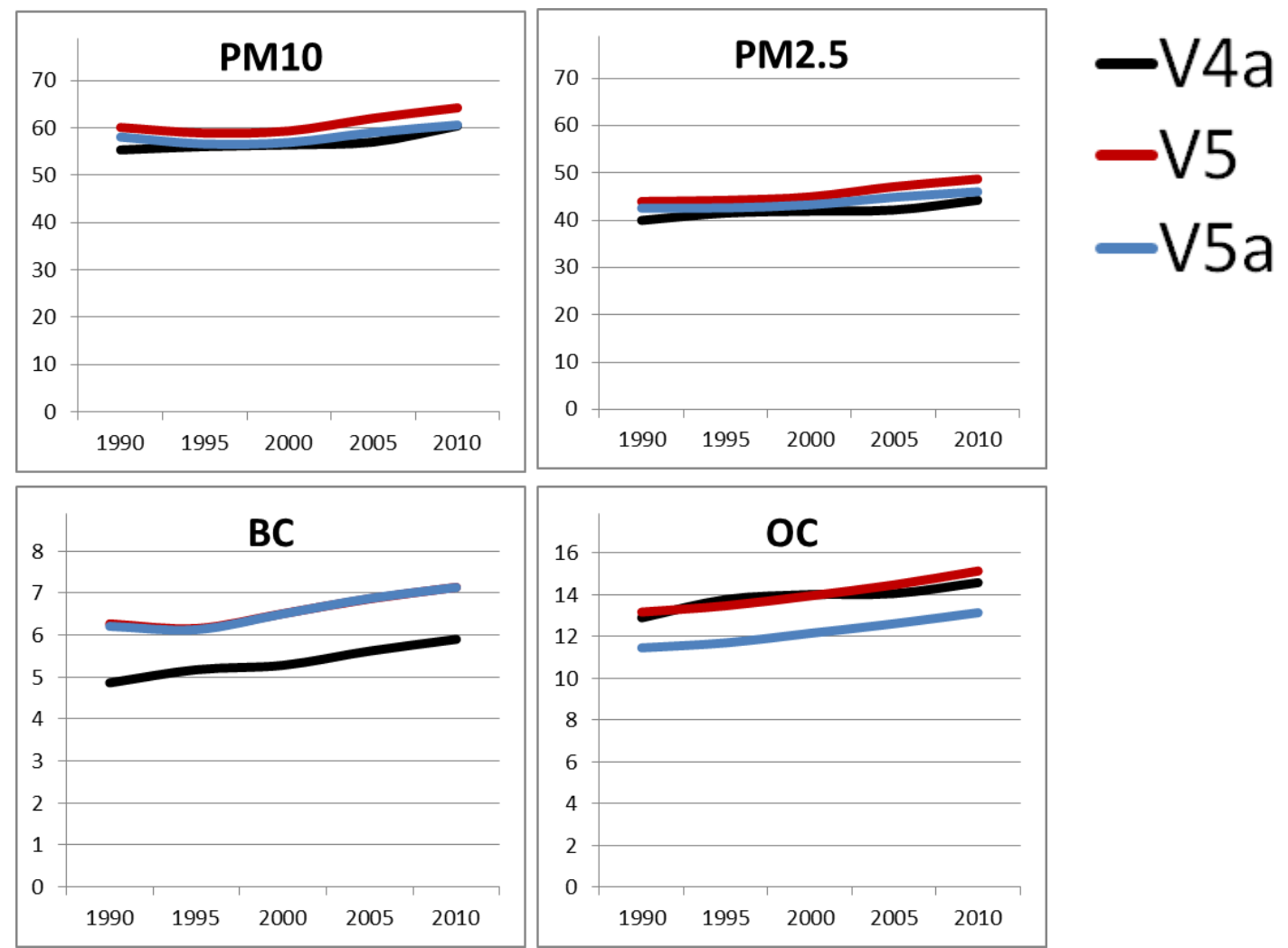

15 Figure S6.1. Global emissions of PM (excluding international shipping and open biomass burning) in the period 1990-2010 in different ECLIPSE scenarios; unit [Tg year $\left.{ }^{-1}\right]$ 
Table S6.2: Global anthropogenic (excluding international shipping \& aviation) emissions of PM10 in ECLIPSE V5a; [Gg year ${ }^{-1}$ ]

\begin{tabular}{|c|c|c|c|c|c|}
\hline Region & 1990 & 1995 & 2000 & 2005 & 2010 \\
\hline 1 Canada & 333 & 315 & 345 & 337 & 334 \\
\hline 2 USA & 2416 & 2158 & 1954 & 1920 & 1630 \\
\hline 3 Mexico & 643 & 621 & 653 & 574 & 572 \\
\hline 4 Rest Central America & 454 & 455 & 479 & 498 & 516 \\
\hline 5 Brazil & 1228 & 1295 & 1250 & 1385 & 1456 \\
\hline 6 Rest South America & 1018 & 1155 & 1138 & 1131 & 1192 \\
\hline 7 Northern Africa & 1022 & 1152 & 1355 & 1144 & 1194 \\
\hline 8 Other Africa & 4393 & 4993 & 5831 & 6425 & 7150 \\
\hline 10 South Africa & 682 & 738 & 747 & 848 & 818 \\
\hline 11 Western Europe & 3294 & 2458 & 2031 & 1747 & 1577 \\
\hline 12 Central Europe & 2944 & 1608 & 1236 & 1046 & 1038 \\
\hline 13 Turkey & 1007 & 756 & 525 & 477 & 571 \\
\hline 14 Ukraine+ & 1854 & 856 & 679 & 707 & 680 \\
\hline 15 Asia-Stan & 836 & 325 & 303 & 314 & 392 \\
\hline 16 Russia+ & 5833 & 2434 & 2314 & 2316 & 2161 \\
\hline 17 Middle East & 836 & 954 & 1055 & 962 & 996 \\
\hline 18 India & 7828 & 8785 & 8654 & 7952 & 8061 \\
\hline 19 Korea & 1227 & 913 & 844 & 816 & 768 \\
\hline 20 China+ & 14057 & 17612 & 18205 & 21230 & 21976 \\
\hline 21 Southeastern Asia & 2291 & 2855 & 2783 & 2451 & 2526 \\
\hline 22 Indonesia+ & 1383 & 1576 & 1673 & 1768 & 1902 \\
\hline 23 Japan & 545 & 435 & 354 & 319 & 267 \\
\hline 24 Oceania & 295 & 303 & 354 & 354 & 342 \\
\hline 25 Rest South Asia & 1695 & 1894 & 2211 & 2349 & 2533 \\
\hline Global & 58112 & 56646 & 56974 & 59071 & 60651 \\
\hline
\end{tabular}


Table S6.3: Global anthropogenic (excluding international shipping \& aviation) emissions of PM2.5 in ECLIPSE V5a; [Gg year ${ }^{-1}$ ]

\begin{tabular}{|c|c|c|c|c|c|}
\hline Region & 1990 & 1995 & 2000 & 2005 & 2010 \\
\hline 1 Canada & 252 & 244 & 250 & 242 & 241 \\
\hline 2 USA & 1629 & 1482 & 1296 & 1275 & 1027 \\
\hline 3 Mexico & 495 & 498 & 526 & 459 & 454 \\
\hline 4 Rest Central America & 395 & 394 & 416 & 428 & 446 \\
\hline 5 Brazil & 938 & 974 & 933 & 1054 & 1098 \\
\hline 6 Rest South America & 825 & 933 & 909 & 901 & 949 \\
\hline 7 Northern Africa & 762 & 852 & 982 & 847 & 909 \\
\hline 8 Other Africa & 4056 & 4606 & 5308 & 5887 & 6575 \\
\hline 10 South Africa & 408 & 444 & 431 & 501 & 490 \\
\hline 11 Western Europe & 2125 & 1700 & 1360 & 1157 & 1037 \\
\hline 12 Central Europe & 1610 & 1020 & 843 & 752 & 775 \\
\hline 13 Turkey & 585 & 480 & 388 & 356 & 425 \\
\hline 14 Ukraine+ & 1072 & 531 & 464 & 483 & 455 \\
\hline 15 Asia-Stan & 562 & 222 & 211 & 222 & 283 \\
\hline 16 Russia+ & 3702 & 1614 & 1530 & 1495 & 1413 \\
\hline 17 Middle East & 686 & 778 & 845 & 784 & 794 \\
\hline 18 India & 5768 & 6453 & 6472 & 5957 & 6032 \\
\hline 19 Korea & 784 & 600 & 547 & 565 & 529 \\
\hline 20 Chinat & 10863 & 13072 & 13633 & 15673 & 16096 \\
\hline 21 Southeastern Asia & 1878 & 2257 & 2198 & 1974 & 2012 \\
\hline 22 Indonesia+ & 1230 & 1371 & 1447 & 1510 & 1604 \\
\hline 23 Japan & 337 & 295 & 236 & 203 & 160 \\
\hline 24 Oceania & 188 & 193 & 210 & 201 & 188 \\
\hline 25 Rest South Asia & 1455 & 1629 & 1859 & 1962 & 2065 \\
\hline Global & 42606 & 42640 & 43294 & 44888 & 46055 \\
\hline
\end{tabular}


Table S6.4: Global anthropogenic (excluding international shipping \& aviation) emissions of PM1 in ECLIPSE V5a; [Gg year ${ }^{-1}$ ]

\begin{tabular}{|c|c|c|c|c|c|}
\hline Region & 1990 & 1995 & 2000 & 2005 & 2010 \\
\hline 1 Canada & 184 & 195 & 196 & 187 & 190 \\
\hline 2 USA & 1163 & 1095 & 949 & 930 & 727 \\
\hline 3 Mexico & 375 & 378 & 395 & 361 & 357 \\
\hline 4 Rest Central America & 329 & 331 & 353 & 366 & 390 \\
\hline 5 Brazil & 706 & 720 & 718 & 819 & 846 \\
\hline 6 Rest South America & 657 & 732 & 708 & 712 & 764 \\
\hline 7 Northern Africa & 447 & 476 & 514 & 485 & 542 \\
\hline 8 Other Africa & 3724 & 4213 & 4838 & 5416 & 6064 \\
\hline 10 South Africa & 285 & 309 & 307 & 354 & 354 \\
\hline 11 Western Europe & 1397 & 1171 & 966 & 834 & 751 \\
\hline 12 Central Europe & 894 & 667 & 619 & 579 & 607 \\
\hline 13 Turkey & 386 & 341 & 286 & 263 & 311 \\
\hline 14 Ukraine+ & 565 & 325 & 279 & 278 & 261 \\
\hline 15 Asia-Stan & 292 & 154 & 146 & 154 & 198 \\
\hline 16 Russia+ & 1988 & 1078 & 1011 & 936 & 852 \\
\hline 17 Middle East & 501 & 562 & 596 & 614 & 615 \\
\hline 18 India & 4500 & 4992 & 5016 & 4700 & 5031 \\
\hline 19 Korea & 635 & 510 & 450 & 464 & 429 \\
\hline 20 China+ & 9153 & 11251 & 11731 & 12473 & 11606 \\
\hline 21 Southeastern Asia & 1800 & 2204 & 2093 & 1791 & 1803 \\
\hline 22 Indonesia+ & 1135 & 1254 & 1315 & 1373 & 1453 \\
\hline 23 Japan & 258 & 229 & 157 & 126 & 87 \\
\hline 24 Oceania & 140 & 146 & 155 & 143 & 133 \\
\hline 25 Rest South Asia & 1303 & 1445 & 1625 & 1714 & 1811 \\
\hline Global & 32816 & 34780 & 35422 & 36073 & 36180 \\
\hline
\end{tabular}


Table S6.5: Global anthropogenic (excluding international shipping \& aviation) emissions of BC in ECLIPSE V5a; [Gg year ${ }^{-1}$ ]

\begin{tabular}{|c|c|c|c|c|c|}
\hline Region & 1990 & 1995 & 2000 & 2005 & 2010 \\
\hline 1 Canada & 44 & 49 & 51 & 49 & 49 \\
\hline 2 USA & 311 & 291 & 281 & 279 & 201 \\
\hline 3 Mexico & 76 & 77 & 82 & 84 & 88 \\
\hline 4 Rest Central America & 52 & 54 & 61 & 65 & 71 \\
\hline 5 Brazil & 143 & 148 & 160 & 171 & 179 \\
\hline 6 Rest South America & 115 & 135 & 140 & 150 & 169 \\
\hline 7 Northern Africa & 127 & 120 & 117 & 121 & 140 \\
\hline 8 Other Africa & 752 & 836 & 942 & 1030 & 1135 \\
\hline 10 South Africa & 57 & 59 & 57 & 74 & 72 \\
\hline 11 Western Europe & 331 & 335 & 307 & 287 & 246 \\
\hline 12 Central Europe & 126 & 112 & 112 & 121 & 134 \\
\hline 13 Turkey & 60 & 59 & 53 & 51 & 67 \\
\hline 14 Ukraine+ & 88 & 59 & 45 & 41 & 36 \\
\hline 15 Asia-Stan & 50 & 28 & 33 & 38 & 55 \\
\hline 16 Russiat & 439 & 251 & 238 & 226 & 177 \\
\hline 17 Middle East & 174 & 183 & 210 & 243 & 262 \\
\hline 18 India & 853 & 931 & 884 & 908 & 1022 \\
\hline 19 Korea & 135 & 84 & 71 & 84 & 74 \\
\hline 20 Chinat & 1348 & 1347 & 1655 & 1823 & 1924 \\
\hline 21 Southeastern Asia & 300 & 299 & 304 & 328 & 333 \\
\hline 22 Indonesiat & 243 & 260 & 275 & 279 & 290 \\
\hline 23 Japan & 67 & 74 & 66 & 50 & 29 \\
\hline 24 Oceania & 30 & 32 & 35 & 35 & 33 \\
\hline 25 Rest South Asia & 288 & 304 & 325 & 337 & 348 \\
\hline Global & 6210 & 6129 & 6505 & 6872 & 7134 \\
\hline
\end{tabular}


Table S6.6: Global anthropogenic (excluding international shipping \& aviation) emissions of OC in ECLIPSE V5a; [Gg year ${ }^{-1}$ ]

\begin{tabular}{|c|c|c|c|c|c|}
\hline Region & 1990 & 1995 & 2000 & 2005 & 2010 \\
\hline 1 Canada & 72 & 77 & 77 & 72 & 74 \\
\hline 2 USA & 448 & 434 & 388 & 379 & 308 \\
\hline 3 Mexico & 162 & 162 & 164 & 158 & 155 \\
\hline 4 Rest Central America & 144 & 149 & 159 & 169 & 181 \\
\hline 5 Brazil & 251 & 258 & 275 & 311 & 314 \\
\hline 6 Rest South America & 297 & 329 & 315 & 324 & 344 \\
\hline 7 Northern Africa & 145 & 150 & 155 & 166 & 192 \\
\hline 8 Other Africa & 1627 & 1842 & 2124 & 2408 & 2701 \\
\hline 10 South Africa & 101 & 108 & 110 & 129 & 130 \\
\hline 11 Western Europe & 495 & 422 & 343 & 284 & 253 \\
\hline 12 Central Europe & 224 & 201 & 217 & 220 & 234 \\
\hline 13 Turkey & 114 & 108 & 95 & 88 & 107 \\
\hline 14 Ukraine+ & 149 & 102 & 82 & 77 & 72 \\
\hline 15 Asia-Stan & 90 & 66 & 62 & 64 & 86 \\
\hline 16 Russiat & 509 & 332 & 304 & 256 & 231 \\
\hline 17 Middle East & 190 & 217 & 220 & 237 & 229 \\
\hline 18 India & 1530 & 1623 & 1596 & 1630 & 1755 \\
\hline 19 Korea & 200 & 157 & 147 & 157 & 148 \\
\hline 20 Chinat & 3147 & 3264 & 3500 & 3564 & 3599 \\
\hline 21 Southeastern Asia & 526 & 548 & 567 & 598 & 632 \\
\hline 22 Indonesia+ & 431 & 473 & 514 & 551 & 595 \\
\hline 23 Japan & 51 & 54 & 49 & 40 & 29 \\
\hline 24 Oceania & 52 & 55 & 57 & 51 & 46 \\
\hline 25 Rest South Asia & 502 & 562 & 628 & 680 & 726 \\
\hline Global & 11456 & 11695 & 12150 & 12610 & 13140 \\
\hline
\end{tabular}




\section{S7 Regional resolution}

The spatial resolution of the GAINS model is discussed section 2.4 of the paper and the list of all 170 regions can be obtained from the online model. In principle, GAINS distinguishes single countries in Europe (exception in Russia for which European and Asian part is included separately) North America, Australia and New Zealand, for Asia several larger

5 countries are divided into provinces or states (larger administrative units in, e.g., China, India, Indonesia, Japan, etc.) while Middle East represented as one region or (most recent versions) distinguishes Iran, Saudi Arabia, Israel, and the rest of Middle East. Africa is divided into four regions: South Africa, Egypt, North Africa, and other Africa. Latin America and Caribbean includes now 13 regions with all larger countries treated separately while Central America as well as Caribbean states are grouped in two regions. While such resolution of 170 regions is used for the calculation of emissions, the

10 presentation of data and results differs between the on-line models available for specific world regions, e.g., for Europe and Asia the full resolution is available, while in the global model application (http://magcat.iiasa.ac.at/gains/IAM/index.login) the data and results are presented for 25 regions (Fig. S7.1). This follows closely the IMAGE model ${ }^{1}$ resolution; often used or compatible with several global integrated assessment models.

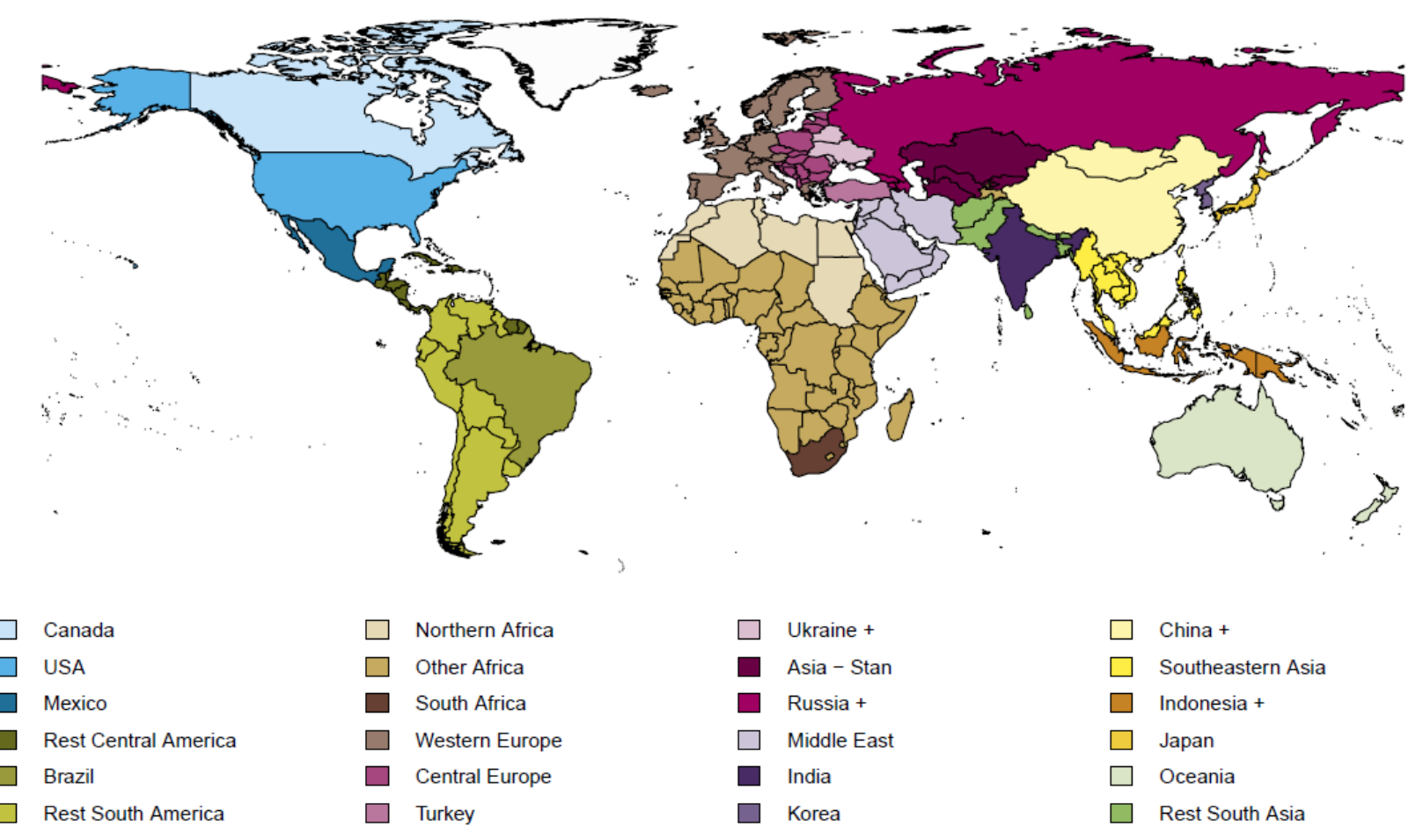

15 Figure S7.1. Regions distinguished in the global GAINS online application.

\footnotetext{
${ }^{1}$ http://themasites.pbl.nl/models/image/index.php/Region_classification_map
} 


\section{S8 Sectoral resolution}

Table S8.1: Source sector resolution in the GAINS model for calculation of PM emissions

\begin{tabular}{lll}
\hline Key source category & Source sectors & Fuel category or activity type \\
\hline \multirow{2}{*}{ Energy sector } & & \\
& $\begin{array}{l}\text { Power plants (distinguishing small, large, old, new plants); } \\
\text { Diesel generators; } \\
\end{array}$ & Cxtraction and distribution of solid and liquid fuels (fugitive as as, biomass, waste \\
& well as combustion from gas flaring) & Coal, oil \\
& Briquette production & Production \\
\hline
\end{tabular}

Residential combustion

Cooking stoves; Heating (distinguishing fireplaces, stoves, house

Coal, fuelwood, dung, oil, gas, boilers, mid-size residential boilers)

Kerosene lighting agricultural residues, charcoal

Waste (trash) burning Kerosene

Waste

Industrial combustion

Iron and Steel; Pulp and Paper; Chemical; Non-ferrous metals; Coal, oil, gas, biomass, waste Non-metallic minerals (excl. Bricks); Other

Industrial processes

Iron and steel industry divided into: Pig iron; Coke ovens;

Production

Agglomeration plants - pellets; Agglomeration plants - sinter;

Open hearth; Electric Arc; Basic oxygen; Rolling mills; Cast Iron

Non-ferrous metals (copper and nickel smelters); Primary

Production

aluminium; Secondary aluminium; Cement; Lime; Carbon black

production; Glass production; Mineral fertilizer production; Brick

manufacturing; Pulp and paper

Refineries

Handling and storage of bulk industrial and agricultural products (fugitive)

Crude oil throughput

Million tons of products

Road transport

Passenger cars and vans; Light duty vehicles; Heavy duty

vehicles; Busses; Motorcycles (4-stroke); Mopeds (2-stroke)

Gasoline, diesel, CNG, LPG, $\mathrm{km}$ driven (for calculation of non-exhaust emissions)

Non-road transport

Agricultural and forestry; Construction and mining; Railways; Inland navigation; Coastal shipping; Aviation (landing and take-

Diesel, gasoline, CNG, jet fuel off); 2-stroke engines (e.g., in household, forestry, etc.); Other and kerosene, heavy fuel oil,

land based machinery

coal

Agriculture

Arable land operations

Arable land area

Livestock housing

Open burning of agricultural waste

Cattle, pigs, poultry

Waste burned

Other

Fireworks; Cigarette smoking; Barbeques; Cremation

Population

Construction (fugitive)

Constructed area 


\section{S8 Comparison of regional estimates with selected studies}

The table S8.1 provides ECLIPSE V5a PM estimates for selected regions and years (from the period 2000-2010) and compares them with selected regional peer-reviewed studies.

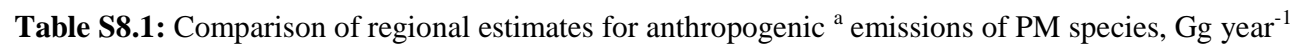

\begin{tabular}{|c|c|c|c|c|c|}
\hline Region $-($ Source $)-$ Year & PM10 & PM2.5 & PM1 & $\mathrm{BC}$ & $\mathrm{OC}$ \\
\hline \multicolumn{6}{|l|}{ Global } \\
\hline This study - 1995 & 57830 & 43762 & 35902 & 6206 & 11949 \\
\hline (Bond et al., 2004) - 1996 & & & & 4997 & 10481 \\
\hline This study - 2000 & 58366 & 44613 & 36741 & 6595 & 12449 \\
\hline (Bond et al., 2013) - 2000 & & & & 4870 & \\
\hline This study - 2010 & 62537 & 47843 & 37819 & 7264 & 13548 \\
\hline HTAP_v2 (Janssens-Maenhout et al., 2015) - 2010 & 50292 & 32761 & & 5525 & 13581 \\
\hline \multicolumn{6}{|l|}{ China } \\
\hline This study - 2000 & 18061 & 13554 & 11685 & 1646 & 3487 \\
\hline (Cao et al., 2006) - 2000 & & & & 1496 & 4211 \\
\hline (Streets et al., 2003) - 2000 & & & & 1049 & 3385 \\
\hline (Klimont et al., 2009) - 2000 & & & & 1345 & 3205 \\
\hline (Lu et al., 2011) - 2000 & & & & 1244 & 2823 \\
\hline (Ohara et al., 2007) - 2000 & & & & 1093 & 2563 \\
\hline (Bond et al., 2013) - 2000 & & & & $1200^{\mathrm{b}}$ & $2800^{\mathrm{b}}$ \\
\hline (Zhang et al., 2006) - 2001 & 17120 & 12100 & & & \\
\hline This study - 2005 & 21087 & 15593 & 12428 & 1813 & 3552 \\
\hline (Zhang et al., 2009) - 2006 & 18223 & 13266 & & 1811 & 3217 \\
\hline (Klimont et al., 2009) - 2005 & & & & 1366 & 2812 \\
\hline This study - 2010 & 21827 & 16019 & 11564 & 1915 & 3589 \\
\hline (Lu et al., 2011) - 2010 & & & & 1838 & 3907 \\
\hline (Kurokawa et al., 2013) - 2008 & 21606 & 14514 & & 1589 & 3081 \\
\hline (Guan et al., 2014) - 2010 & & 12100 & & & \\
\hline HTAP_v2 (Janssens-Maenhout et al., 2015) - 2010 & 16615 & 12199 & & 1764 & 3384 \\
\hline (Kondo et al., 2011) - 2008 & & & & 1940 & \\
\hline \multicolumn{6}{|l|}{ India } \\
\hline This study, 2000 & 8654 & 6472 & 5016 & 884 & 1596 \\
\hline (Streets et al., 2003) - 2000 & & & & 600 & 2837 \\
\hline (Ohara et al., 2007) - 2000 & & & & 795 & 3268 \\
\hline (Klimont et al., 2009) - 2000 & & & & 842 & 1887 \\
\hline (Lu et al., 2011) - 2000 & & & & 736 & 1990 \\
\hline (Bond et al., 2013) - /2000 & & & & $500^{\mathrm{b}}$ & $1600^{b}$ \\
\hline
\end{tabular}




\begin{tabular}{|c|c|c|c|c|c|}
\hline Region $-($ Source $)-$ Year & PM10 & PM2.5 & PM1 & $\mathrm{BC}$ & $\mathrm{OC}$ \\
\hline (Reddy and Venkataraman, 2002a, 2002b) - 1998-99 & & 4300 & & 380 & 1250 \\
\hline This study, 2005 & 7952 & 5957 & 4700 & 908 & 1630 \\
\hline (Zhang et al., 2009) - 2006 & 4002 & 3111 & & 344 & 888 \\
\hline (Klimont et al., 2009) - 2005 & & & & 1029 & 2132 \\
\hline This study, 2010 & 8061 & 6032 & 5091 & 1022 & 1755 \\
\hline (Lu et al., 2011) - 2010 & & & & 996 & 2582 \\
\hline HTAP_v2 (Janssens-Maenhout et al., 2015) - 2010 & 8280 & 6230 & & 1019 & 2530 \\
\hline (Kurokawa et al., 2013) - 2008 & 6651 & 4884 & & 713 & 2286 \\
\hline \multicolumn{6}{|l|}{ Europe $^{\mathrm{c}}$} \\
\hline This study - 1995 & 6905 & 4584 & 3071 & 675 & 1021 \\
\hline (Kupiainen and Klimont, 2007) - 1995 & & & & 717 & 1053 \\
\hline (Schaap et al., 2004) - 1995 & & & & 760 & \\
\hline (Bond et al., 2004) - 1996 & & & & 678 & 947 \\
\hline This study - 2000 & 5579 & 3843 & 2668 & 618 & 910 \\
\hline (Kupiainen and Klimont, 2007) - 2000 & & & & 680 & 996 \\
\hline (Kupiainen and Klimont, 2004) - 2000 & & & 2772 & 672 & 988 \\
\hline This study - 2010 & 5008 & 3471 & 2393 & 562 & 806 \\
\hline HTAP_v2 (Janssens-Maenhout et al., 2015) ${ }^{\mathrm{d}}-2010$ & 2951 & 2133 & & 382 & 638 \\
\hline LRTAP reporting (www.ceip.at) - 2010 & 4784 & 3250 & & & \\
\hline \multicolumn{6}{|l|}{ Russian Federation } \\
\hline This study - 2010 & 2108 & 1368 & 815 & 170 & 213 \\
\hline HTAP_v2 (Janssens-Maenhout et al., 2015) - 2010 & 562 & 313 & & 60 & 42 \\
\hline (Huang et al., 2015) - 2010 & & & & 224 & \\
\hline \multicolumn{6}{|l|}{ Russian Federation - European part only } \\
\hline This study - 2010 & 1090 & 734 & 427 & 71 & 122 \\
\hline LRTAP reporting (www.ceip.at) - 2010 & 569 & 367 & & & \\
\hline \multicolumn{6}{|l|}{ US } \\
\hline This study - 2000 & 1954 & 1296 & 949 & 289 & 388 \\
\hline (Battye et al., 2002) - 1999 & & & & 430 & \\
\hline (Reff et al., 2009) - 2000 & & & & 440 & 960 \\
\hline (Bond et al., 2013) - 2000 & & & & $350^{\mathrm{b}}$ & $500^{\mathrm{b}}$ \\
\hline This study - 2010 & 1630 & 1027 & 727 & 201 & 308 \\
\hline$\left(\right.$ US EPA, 2011) ${ }^{\mathrm{e}}-2011$ & 17597 & 4513 & & $513\left(313^{\mathrm{f}}\right)$ & \\
\hline HTAP_v2 (Janssens-Maenhout et al., 2015) - 2010 & 1973 & 1640 & & 295 & 471 \\
\hline
\end{tabular}

${ }^{a}$ Based on the information available in the quoted studies, all presented estimates exclude forest fires but include agricultural burning, unless stated otherwise; ${ }^{b}$ Excluding agricultural burning; ${ }^{\mathrm{c}}$ Includes European part of Russian Federation (except HTAP_v2);

${ }^{\mathrm{d}}$ Excluding any territories of Russian Federation; ${ }^{\mathrm{e}}$ Including wildfires and prescribed burning; ${ }^{\mathrm{f}}$ Excluding wildfires and prescribed burning 


\section{References}

AIT: Small and Medium scale Industries in Asia: Energy and Environment. Brick and Ceramic Sectors, Regional Energy Resources Information Center (RERIC), Asian Institute of Technology (AIT), Pathumthani, Thailand., 2003.

Alam, S. A.: Use of biomass fuels in the brick-making industries of Sudan: Implications for deforestation and greenhouse 5 gas emission, M. Sc., University of Helsinki, Helsinki, Finland, April., 2006.

Alves, C., Gonçalves, C., Fernandes, A. P., Tarelho, L. and Pio, C.: Fireplace and woodstove fine particle emissions from combustion of western Mediterranean wood types, Atmospheric Research, 101(3), 692-700, doi:10.1016/j.atmosres.2011.04.015, 2011.

Amann, M., Bertok, I., Borken-Kleefeld, J., Cofala, J., Heyes, C., Hoglund-Isaksson, L., Kiesewetter, G., Klimont, Z.,

10 Schöpp, W., Vellinga, N. and Winiwarter, W.: Adjusted historic emission data, projections, and optimized emission reduction targets for 2030 - A comparison with COM data 2013, IIASA, Laxenburg, Austria. [online] Available from: http://ec.europa.eu/environment/air/pdf/review/TSAP_16a.pdf, 2015.

Anayochukwu, A. V., Nnene, E. A. and Onyeka, A. E.: Assessment of Environmental Impact of Power Generation in Banking Industry, Journal of Energy, Environment \& Carbon Credits, 3(1), 13-21, 2013.

15 BASIN: Vertical Shaft Brick Kiln - Technology transfer Indian experience - 2, Case Study, Building Advisory Aervice and Information Network (BASIN), Eschborn, Germany. [online] Available from: http://www.gtz.de/basin, 1998.

BASIN: VSBK-Newsletter 3, Newsletter, Building Advisory Aervice and Information Network (BASIN), Eschborn, Germany. [online] Available from: http://www.gtz.de/basin, 1999.

Battye, W., Boyer, K. and Pace, T. G.: Methods for improving global inventories of black carbon and organic carbon 20 particulates, EC/R Inc., Chapel Hill, North Carolina, US., 2002.

Bellprat, O.: Brick Kiln Evaluation Study in the Bajio Region GTO, Mexico., INE, ETH., 2009.

Boman, C., Nordin, A., Pettersson, E. and Wiinikka, H.: State-of-the-art of small-scale biomass combustion with respect to fine particle emissions - country report from Sweden, Graz, Austria., 2008.

Boman, C., Pettersson, E., Westerholm, R., Boström, D. and Nordin, A.: Stove Performance and Emission Characteristics in

25 Residential Wood Log and Pellet Combustion, Part 1: Pellet Stoves, Energy Fuels, 25(1), 307-314, doi:10.1021/ef100774x, 2011.

Bond, T. C., Streets, D. G., Yarber, K. F., Nelson, S. M., Woo, J. H. and Klimont, Z.: A technology-based global inventory of black and organic carbon emissions from combustion, J. Geophys. Res., 109(D14), 1-43, doi:10.1029/2003JD003697, 2004.

30 Bond, T. C., Doherty, S. J., Fahey, D. W., Forster, P. M., Berntsen, T., DeAngelo, B. J., Flanner, M. G., Ghan, S., Kärcher, B., Koch, D., Kinne, S., Kondo, Y., Quinn, P. K., Sarofim, M. C., Schultz, M. G., Schulz, M., Venkataraman, C., Zhang, H., Zhang, S., Bellouin, N., Guttikunda, S. K., Hopke, P. K., Jacobson, M. Z., Kaiser, J. W., Klimont, Z., Lohmann, U., Schwarz, J. P., Shindell, D., Storelvmo, T., Warren, S. G. and Zender, C. S.: Bounding the role of black carbon in the climate system: A scientific assessment, Journal of Geophysical Research: Atmospheres, 118, 5380-5552, doi:10.1002/jgrd.50171, 2013. 
Cao, G., Zhang, X. and Zheng, F.: Inventory of black carbon and organic carbon emissions from China, Atmospheric Environment, 40(34), 6516-6527, doi:10.1016/j.atmosenv.2006.05.070, 2006.

Chen, Y., Zhi, G., Feng, Y., Liu, D., Zhang, G., Li, J., Sheng, G. and Fu, J.: Measurements of Black and Organic Carbon Emission Factors for Household Coal Combustion in China: Implication for Emission Reduction, Environ. Sci. Technol., 5 43(24), 9495-9500, doi:10.1021/es9021766, 2009.

Cheung, K. L., Polidori, A., Ntziachristos, L., Tzamkiozis, T., Samaras, Z., Cassee, F. R., Gerlofs, M. and Sioutas, C.: Chemical characteristics and oxidative potential of particulate matter emissions from gasoline, diesel, and biodiesel cars, Environmental science \& technology, 43(16), 6334-6340, 2009.

Co, H. X., Dung, N. T., Le, H. A., An, D. D., van Chinh, K. and Oanh, N. T. K.: Integrated management strategies for brick

Croitoru, L. and Sarraf, M.: Benefits and Costs of the Informal Sector: The Case of Brick Kilns in Bangladesh, Journal of Environmental Protection, 3, 476-484, doi:http://dx.doi.org/10.4236/jep.2012.36058, 2012.

Dincer, F. and Elbir, T.: Estimating national exhaust emissions from railway vehicles in Turkey, Science of The Total Environment, 374(1), 127-134, doi:10.1016/j.scitotenv.2006.12.043, 2007.

15 Dwyer, H., Ayala, A., Zhang, S., Collins, J., Huai, T., Herner, J. and Chau, W.: A study of emissions from a Euro 4 light duty diesel vehicle with the European particulate measurement programme, Atmospheric Environment, 44(29), 3469-3476, doi:10.1016/j.atmosenv.2010.06.028, 2010.

EC-JRC/PBL: Emission Database for Global Atmospheric Research (EDGAR)., European Commission (EC), Joint Research Centre (JRC)/Netherlands Environmental Assessment Agency (PBL). [online] Available from: http://edgar.jrc.ec.europa.eu, 202010.

EEA: EMEP/EEA air pollutant emission inventory guidebook, Technical report, European Environmental Agency (EEA), Copenhagen, Denmark. [online] Available from: http://www.eea.europa.eu/publications/emep-eea-guidebook-2013, 2013.

EELA: Caracterizacion de los hornos usados en la industria ladrillera - Proyecto Colombia, Programa Efficiencia Energetica en Ladrillras Artesanales (EELA)., 2011.

25 Erbe, S. O.: Technical, economical and organization analysis of informal brick production in Tercera Chica, SLP, Mexico, Master of Science, Universidad Autonoma de San Luis Potosi, San Luis Potosi, Mexico, February., 2011.

FAO: Status and development issues of the brick industry in Asia, Field Document, FAO Regional Wood Energy Development Programme in Asia, Bangkok, Thailand., 1993.

Fine, P. M., Cass, G. R. and Simoneit, B. R. T.: Chemical characterization of fine particle emissions from the wood stove combustion of prevalent United States tree species, Environ. Eng. Sci., 21(6), 705-721, 2004.

Frey, A. K., Saarnio, K., Lamberg, H., Mylläri, F., Karjalainen, P., Teinilä, K., Carbone, S., Tissari, J., Niemelä, V., Häyrinen, A., Rautiainen, J., Kytömäki, J., Artaxo, P., Virkkula, A., Pirjola, L., Rönkkö, T., Keskinen, J., Jokiniemi, J. and Hillamo, R.: Optical and Chemical Characterization of Aerosols Emitted from Coal, Heavy and Light Fuel Oil, and SmallScale Wood Combustion, Environ. Sci. Technol., 48(1), 827-836, doi:10.1021/es4028698, 2014. 
Gaegauf, C. K., Schmid, M. R. and Güntert, P.: Elemental and Organic Carbon in Flue Gas Particles of Various Wood Combustion Systems, Ökozentrum Langenbruck, Langenbruck, Switzerland. [online] Available from: www.oekozentrum.ch, 2005.

Galvis, B., Bergin, M. and Russell, A.: Fuel-based fine particulate and black carbon emission factors from a railyard area in 5 Atlanta, Journal of the Air \& Waste Management Association, 63(6), 648-658, doi:10.1080/10962247.2013.776507, 2013.

Geller, M. D., Ntziachristos, L., Mamakos, A., Samaras, Z., Schmitz, D. A., Froines, J. R. and Sioutas, C.: Physicochemical and redox characteristics of particulate matter (PM) emitted from gasoline and diesel passenger cars, Atmospheric Environment, 40(36), 6988-7004, doi:10.1016/j.atmosenv.2006.06.018, 2006.

Gilmore, E. A., Lave, L. B. and Adams, P. J.: The costs, air quality and human health effects of meeting peak electricity demand with installed backup generators, Environmental Science \& Technology, 40(22), 6887-6893, 2006.

Gonçalves, C., Alves, C., Evtyugina, M., Mirante, F., Pio, C., Caseiro, A., Schmidl, C., Bauer, H. and Carvalho, F.: Characterisation of PM10 emissions from woodstove combustion of common woods grown in Portugal, Atmospheric Environment, 44(35), 4474-4480, doi:10.1016/j.atmosenv.2010.07.026, 2010.

Gonçalves, C., Evtyugina, M., Alves, C., Monteiro, C., Pio, C. and Tomé, M.: Organic particulate emissions from field 15 burning of garden and agriculture residues, Atmospheric Research, 101(3), 666-680, doi:10.1016/j.atmosres.2011.04.017, 2011.

Graham, L.: Chemical characterization of emissions from advanced technology light-duty vehicles, Atmospheric Environment, 39(13), 2385-2398, doi:10.1016/j.atmosenv.2004.10.049, 2005.

Grieshop, A. P., Boland, D., Reynolds, C. C. O., Gouge, B., Apte, J. S., Rogak, S. N. and Kandlikar, M.: Modeling air 20 pollutant emissions from Indian auto-rickshaws: Model development and implications for fleet emission rate estimates, Atmospheric Environment, 50, 148-156, doi:10.1016/j.atmosenv.2011.12.046, 2012.

Guan, D., Su, X., Zhang, Q., Peters, G. P., Liu, Z., Lei, Y. and He, K.: The socioeconomic drivers of China's primary PM 2.5 emissions, Environmental Research Letters, 9(2), $24010,2014$.

Guttikunda, S. K., Begum, B. A. and Wadud, Z.: Particulate pollution from brick kiln clusters in the Greater Dhaka region,

Bangladesh, Air Quality, Atmosphere \& Health, 6(2), 357-365, doi:10.1007/s11869-012-0187-2, 2013.

Heierli, U. and Maithel, S.: Brick by Brick: The Herculean Task of Cleaning of the Asian Brick Industry, Swiss Agency for Development and Cooperation (SDC), Bern., 2008.

Heierli, U., Jha, H. B. and Gurung, Y.: VSBK \& CESEF Nepal; External Review, 2007.

Herner, J. D., Hu, S., Robertson, W. H., Huai, T., Collins, J. F., Dwyer, H. and Ayala, A.: Effect of Advanced Aftertreatment 30 for PM and NOx Control on Heavy-Duty Diesel Truck Emissions, Environ. Sci. Technol., 43(15), 5928-5933, doi:10.1021/es9008294, 2009.

Huang, K., Fu, J. S., Prikhodko, V. Y., Storey, J. M., Romanov, A., Hodson, E. L., Cresko, J., Morozova, I., Ignatieva, Y. and Cabaniss, J.: Russian anthropogenic black carbon: Emission reconstruction and Arctic black carbon simulation, Journal of Geophysical Research: Atmospheres, 120(21), 11,306-11,333, doi:10.1002/2015JD023358, 2015. 
Jaffe, D. A., Hof, G., Malashanka, S., Putz, J., Thayer, J., Fry, J. L., Ayres, B. and Pierce, J. R.: Diesel particulate matter emission factors and air quality implications from in-service rail in Washington State, USA, Atmospheric Pollution Research, 5(2), 344-351, doi:10.5094/APR.2014.040, 2014.

Janssens-Maenhout, G., Crippa, M., Guizzardi, D., Dentener, F., Muntean, M., Pouliot, G., Keating, T., Zhang, Q., 5 Kurokawa, J., Wankmüller, R., Denier van der Gon, H., Kuenen, J. J. P., Klimont, Z., Frost, G., Darras, S., Koffi, B. and Li, M.: HTAP_v2.2: a mosaic of regional and global emission grid maps for 2008 and 2010 to study hemispheric transport of air pollution, Atmospheric Chemistry and Physics, 15(19), 11411-11432, doi:10.5194/acp-15-11411-2015, 2015.

Johansson, L. S., Leckner, B., Gustavsson, L., Cooper, D., Tullin, C. and Potter, A.: Emission characteristics of modern and old-type residential boilers fired with wood logs and wood pellets, Atmospheric Pollution, 38, 4183-4195, 2004.

10 Johnson, G. R., Jayaratne, E. R., Lau, J., Thomas, V., Juwono, A. M., Kitchen, B. and Morawska, L.: Remote measurement of diesel locomotive emission factors and particle size distributions, Atmospheric Environment, 81, 148-157, doi:10.1016/j.atmosenv.2013.09.019, 2013.

Jordan, T. B. and Seen, A. J.: Effect of airflow setting on the organic composition of woodheater emissions, Environ. Sci. \& Technol., 39(10), 3601-3610, 2005.

15 Just, B., Rogak, S. and Kandlikar, M.: Characterization of Ultrafine Particulate Matter from Traditional and Improved Biomass Cookstoves, Environ. Sci. Technol., 47(7), 3506-3512, doi:10.1021/es304351p, 2013.

Karvosenoja, N.: Emission scenario model for regional air pollution, Monographs of the Boreal Environment Research, 32 [online] Available from: www.enviroment.fi /publications, 2008.

Klimont, Z., Cofala, J., Bertok, I., Amann, M., Heyes, C. and Gyarfas, F.: Modelling Particulate Emissions in Europe. A 20 Framework to Estimate Reduction Potential and Control Costs, Interim Report, International Institute for Applied Systems Analysis (IIASA), Laxenburg, Austria., 2002.

Klimont, Z., Cofala, J., Xing, J., Wei, W., Zhang, C., Wang, S., Kejun, J., Bhandari, P., Mathur, R., Purohit, P., Rafaj, P., Chambers, A., Amann, M. and Hao, J.: Projections of SO2, NOx, and carbonaceous aerosols emissions in Asia, Tellus, 61B(4), 602-617, doi:10.1111/j.1600-0889.2009.00428.x, 2009.

25 Kondo, Y., Oshima, N., Kajino, M., Mikami, R., Moteki, N., Takegawa, N., Verma, R. L., Kajii, Y., Kato, S. and Takami, A.: Emissions of black carbon in East Asia estimated from observations at a remote site in the East China Sea, Journal of Geophysical Research: Atmospheres, 116(D16), n/a-n/a, doi:10.1029/2011JD015637, 2011.

Krasowsky, T., Daher, N., Sioutas, C. and Ban-Weiss, G.: Measurement of particulate matter emissions from in-use locomotives, Atmospheric Environment, 113, 187-196, doi:10.1016/j.atmosenv.2015.04.046, 2015.

30 Kupiainen, K. and Klimont, Z.: Primary Emissions of Submicron and Carbonaceous Particles in Europe and the Potential for their Control, International Institute for Applied Systems Analysis (IIASA), Laxenburg, Austria., 2004.

Kupiainen, K. and Klimont, Z.: Primary emissions of fine carbonaceous particles in Europe, Atmospheric Environment, 41, 2156-2170, doi:10.1016/j.atmosenv.2006.10.066, 2007.

Kurokawa, J., Ohara, T., Morikawa, T., Hanayama, S., Janssens-Maenhout, G., Fukui, T., Kawashima, K. and Akimoto, H.: 35 Emissions of air pollutants and greenhouse gases over Asian regions during 2000-2008: Regional Emission inventory in ASia (REAS) version 2, Atmospheric Chemistry and Physics, 13(21), 11019-11058, doi:10.5194/acp-13-11019-2013, 2013. 
Lamberg, H., Sippula, O., Tissari, J. and Jokiniemi, J.: Effects of Air Staging and Load on Fine-Particle and Gaseous Emissions from a Small-Scale Pellet Boiler, Energy Fuels, 25(11), 4952-4960, doi:10.1021/ef2010578, 2011 a.

Lamberg, H., Nuutinen, K., Tissari, J., Ruusunen, J., Yli-Pirilla, P., Sippula, O., Tapanainen, M., Jalava, P., Makkonen, U. and Teinilla, K.: Physicochemical Characterization of Fine Particles from Small-Scale Wood Combustion, Atmospheric 5 Environment, 45, 7635-7643, doi:10.1016/j. atmosenv.2011.02.072, 2011b.

Li, Q., Li, X., Jiang, J., Duan, L., Ge, S., Zhang, Q., Deng, J., Wang, S. and Hao, J.: Semi-coke briquettes: towards reducing emissions of primary PM2.5, particulate carbon, and carbon monoxide from household coal combustion in China, Scientific Reports, 6, 19306, 2016.

Li, X. H., Wang, S. X., Duan, L., Hao, J. M. and Nie, Y. F.: Carbonaceous aerosol emissions from household biofuel combustion in China., Environ. Sci. Technol., 43, 6076-6081, 2009.

Liu, H., He, K., Lents, J. M., Wang, Q. and Tolvett, S.: Characteristics of Diesel Truck Emission in China Based on Portable Emissions Measurement Systems, Environmental Science \& Technology, 43(24), 9507-9511, doi:10.1021/es902044x, 2009.

Louis, C., Liu, Y., Tassel, P., Perret, P., Chaumond, A. and André, M.: PAH, BTEX, carbonyl compound, black-carbon, $\mathrm{NO} 2$ and ultrafine particle dynamometer bench emissions for Euro 4 and Euro 5 diesel and gasoline passenger cars, 15 Atmospheric Environment, 141, 80-95, doi:10.1016/j.atmosenv.2016.06.055, 2016.

Lu, Z., Zhang, Q. and Streets, D. G.: Sulfur dioxide and primary carbonaceous aerosol emissions in China and India, 19962010, Atmos. Chem. Phys., 11(18), 9839-9864, 2011.

Maithel, S.: Factsheets about Brick Kilns in South and South-East Asia, [online] Available from: http://www.ccacoalition.org/en/resources/factsheets-about-brick-kilns-south-and-south-east-asia, 2014.

20 Maithel, S., Lalchandani, D., Malhotra, G., Bhanware, P., Uma, R., Ragavan, S., Athalye, V., Bindiya, K., Reddy, S., Bond, T. C., Weyant, C., Baum, E., Kim Thoa, V. T., Thu Phuong, N. and Kim Thanh, T.: Brick Kilns Performance Asessment; A Roadmap for Cleaner Brick Production in India, Shakti Sustainable Energy Foundation and Climate Works Foundation. [online] Available from: http://www.catf.us/resources/publications/view/161, 2012.

McDonald, J. D., Zielinska, B., Fujita, E. M., Sagebiel, J. C., Chow, J. C. and Watson, J. G.: Fine Particle and Gaseous 25 Emission Rates from Residential Wood Combustion., Environ. Sci. \& Technol., 34, 2080-2091, 2000.

Ohara, T., Akimoto, H., Kurokawa, J., Horii, N., Yamaji, K., Yan, X. and Hayasaka, T.: An Asian emission inventory of anthropogenic emission sources for the period 1980-2020, Atmos. Chem. Phys., 7, 4419-4444, 2007.

Pettersson, E., Boman, C., Westerholm, R., Boström, D. and Nordin, A.: Stove Performance and Emission Characteristics in Residential Wood Log and Pellet Combustion, Part 2: Wood Stove, Energy Fuels, 25(1), 315-323, doi:10.1021/ef1007787, $30 \quad 2011$

PRAL: Case study. Behind the bricks: An experience in the integrated management of the informal sector, PRAL (Regional Clean Air Program), Lima, Peru., 2012.

Reddy, S. M. and Venkataraman, C.: Inventory of aerosol and sulphur dioxide emissions from India. Part I - Fossil fuel combustion, Atmospheric Environment, 36, 677-697, 2002a.

35 Reddy, S. M. and Venkataraman, C.: Inventory of aerosol and sulphur dioxide emissions from India. Part II - biomass combustion, Atmospheric Environment, 36, 699-712, 2002b. 
Reff, A., Bhave, P. V., Simon, H., Pace, T. G., Pouliot, G. A., Mobley, J. D. and Houyoux, M.: Emissions Inventory of PM2.5 Trace Elements across the United States, Environ. Sci. Technol., 43(15), 5790-5796, doi:10.1021/es802930x, 2009.

Roden, C. A., Bond, T. C., Conway, S., Osorto Pinel, A. B., MacCarty, N. and Still, D.: Laboratory and field investigations of particulate and carbon monoxide emissions from traditional and improved cookstoves, Atmospheric Environment, 43(6),

5 1170-1181, doi:10.1016/j.atmosenv.2008.05.041, 2009.

Rozemuller, B.: An Overview of the Brick and Tile Manufacturing Industry in North West Cambodia, Center for Advanced Studies, UNDP/CARERE, Phnom Penh, Kingdom of Cambodia., 1999.

Samuel Hall Consulting: A Rapid Assessment of Bonded Labour in Brick Kilns in Afghanistan, International Labour Organisation (ILO), Kabul, Afghanistan., 2011.

10 Schaap, M., Van Der Gon, H. A. C. D., Dentener, F. J., Visschedijk, A. J. H., Van Loon, M., ten Brink, H. M., Putaud, J.-P., Guillaume, B., Liousse, C. and Builtjes, P. J. H.: Anthropogenic black carbon and fine aerosol distribution over Europe, Journal of Geophysical Research: Atmospheres, 109(D18), n/a-n/a, doi:10.1029/2003JD004330, 2004.

Schmidl, C., Luisser, M., Padouvas, E., Lasselsberger, L., Rzaca, M., Cruz, C. .-S., Handler, M., Peng, G., Bauer, H. and Puxbaum, H.: Particulate and gaseous emissions from manually and automatically fired small scale combustion systems.,

15 Atmospheric Environment, 45, 7443-7454, 2011.

Scott, G.: Baseline Study for Clamp Kilns in South Africa and Possible Regulatory Approaches, 2013.

Shah, S. D., Cocker III, D. R., Johnson, K. C., Lee, J. M., Soriano, B. L. and Miller, J. W.: Reduction of particulate matter emissions from diesel backup generators equipped with four different exhaust after treatment devices, Environmental Science \& Technology, 41(14), 5070-5076, doi:10.1021/es0614161, 2007.

20 Sippula, O., Hytönen, K., Tissari, J., Raunemaa, T. and Jokiniemi, J.: Effect of Wood Fuel on the Emissions from a TopFeed Pellet Stove, Energy Fuels, 21(2), 1151-1160, doi:10.1021/ef060286e, 2007.

Spezzano, P., Picini, P., Cataldi, D., Messale, F., Manni, C. and Santino, D.: Particle-phase Polycyclic Aromatic Hydrocarbon Emissions from Non-catalysed, In-use Four-stroke Scooters, Environmental Monitoring and Assessment, 133(1), 105-117, doi:10.1007/s10661-006-9564-3, 2007.

25 Spezzano, P., Picini, P., Cataldi, D., Messale, F. and Manni, C.: Particle- and gas-phase emissions of polycyclic aromatic hydrocarbons from two-stroke, 50-cm3 mopeds, Atmospheric Environment, 42(18), 4332-4344, doi:10.1016/j.atmosenv.2008.01.008, 2008.

Stratus Consulting: CCAC initiative to mitigate black carbon and other pollutants from brick production: Regional Assessment. Black Carbon Mitigation in Brick Production: A Summary for Brazil, Chile, Colombia, Mexico, Nigeria, and 30 Peru, Climate and Clean Air Coalition (CCAC)., 2014.

Streets, D. G., Bond, T. C., Carmichael, G. R., Fernandes, S. D., Fu, Q., He, D., Klimont, Z., Nelson, S. M., Tsai, N. Y., Wang, M. Q., Woo, J. H. and Yarber, K. F.: An inventory of gaseous and primary aerosol emissions in Asia in the year 2000, Journal of Geophysical Research, 108(D21), 1-23, doi:10.1029/2002JD003093, 2003.

Subrahmanya, M. H. B.: Labour productivity, energy intensity and economic performance in small enterprises: A study of 35 brick enterprises cluster in India, Energy Conversion and Management, 47, 763-777, doi:10.1016/j.enconman.2005.05.021, 2006. 
Subramanian, R., Winijkul, E., Bond, T. C., Thiansathit, W., Oanh, N. T. K., Paw-armart, I. and Duleep, K. G.: ClimateRelevant Properties of Diesel Particulate Emissions: Results from a Piggyback Study in Bangkok, Thailand, Environmental Science \& Technology, 43(11), 4213-4218, doi:10.1021/es8032296, 2009.

SwissContact: Report on comparative portfolio of technologies and practices in Asia and LAC; Assessment of Gaps., 5 CCAC; Brick Production Initiative., 2014a.

SwissContact: Report on identified past work on effective policies., CCAC; Brick Production Initiative., 2014b.

SwissContact: Survey of the Status of the Informal Clay Brick Making Sector; Eastern Cape, South Africa, 2014, 2014c.

Tang, N. W., Apte, J. S., Martien, P. T. and Kirchstetter, T. W.: Measurement of black carbon emissions from in-use dieselelectric passenger locomotives in California, Atmospheric Environment, 115, 295-303, 10 doi:10.1016/j.atmosenv.2015.05.001, 2015.

Tissari, J., Sippula, O., Kouki, J., Vuorio, K. and Jokiniemi, J.: Fine Particle and Gas Emissions from the Combustion of Agricultural Fuels Fired in a 20 kW Burner, Energy Fuels, 22(3), 2033-2042, doi:10.1021/ef700766y, 2008.

Tsai, J. H., Chen, S. J., Huang, K. L., Lin, Y. C., Lee, W. J., Lin, C. C. and Lin, W. Y.: PM, carbon, and PAH emissions from a diesel generator fuelled with soy-biodiesel blends, Journal of Hazardous Materials, 179(1-3), 237-243, 15 doi:10.1016/j.jhazmat.2010.02.085, 2010.

Uma, R., Kandpal, T. C. and Kishore, V. V. N.: Emission characteristics of an electricity generation system in diesel alone and dual fuel modes, Biomass and Bioenergy, 27(2), 195-203, doi:10.1016/j.biombioe.2004.01.003, 2004.

UNEP/WMO: Integrated Assessment of Black Carbon and Tropospheric Ozone, Nairobi, Kenya. [online] Available from: www.unep.org/dewa/Portals/67/pdf/BlackCarbon_report.pdf, 2011.

20 US EPA: 2011 Version 6 Air Emissions Modeling Platforms, [online] Available from: https://www.epa.gov/air-emissionsmodeling/2011-version-6-air-emissions-modeling-platforms, 2011.

Verma, S. and Uppal, J.: Use of biomass in brick kilns, Ministry of New and Renewable Energy, Government of India, 6(4), 24-27, 2013.

Wang, A., Ge, Y., Tan, J., Fu, M., Shah, A. N., Ding, Y., Zhao, H. and Liang, B.: On-road pollutant emission and fuel consumption characteristics of buses in Beijing, J. Env. Sci., 23(3), 419-426, doi:10.1016/S1001-0742(10)60426-3, 2011.

Wang, S.-X., Zhao, X.-J., Li, X.-H., Wei, W. and Hao, J.-M.: Emission characteristics of fine particles from grate firing boilers, Environmental Science, 30(4), 963-968, 2009.

Watson, J. G., Miller, W., Moosmuller, H., Kuhns, H. D., Chow, J. C., Zhu, D. and Cocker, D. R.: Characterization of OffRoad Diesel Emissions of Criteria Pollutants, CY2006 Annual Report, WP-1336, Desert Research Institute, Reno, NV., 2006.

Watson, J. G., Miller, J. W., Moosmüller, H., Kuhns, H. D., Chow, J. C., Chang, O., Nussbaum, N., Mazzoleni, C., Zhu, D., Barber, P. W., Kemme, M. R. and Cocker, D. R.: Characterization of off-road diesel emissions of criteria pollutants, Desert Research Institute, Reno, Nonline] Available from: https://www.dri.edu/images/stories/editors/eafeditor/Watsonetal2008SERDPFinalReportDieselWP1366.pdf, 2008. 
Weyant, C., Athalye, V., Ragavan, S., Rajarathnam, U., Lalchandani, D., Maithel, S., Baum, E. and Bond, T. C.: Emissions from South Asian Brick Production, Environ. Sci. Technol., 48(11), 6477-6483, doi:10.1021/es500186g, 2014.

World Bank: Introducing energy-efficient clean technologies in the brick sector of Bangladesh, World Bank, Washington DC, USA. [online] Available from: http://documents.worldbank.org/curated/en/2011/06/15492357/introducing-energy5 efficient-clean-technologies-brick-sector-bangladesh, 2011.

Yang, H.-H., Hsieh, L.-T., Liu, H.-C. and Mi, H.-H.: Polycyclic aromatic hydrocarbon emissions from motorcycles, Atmospheric Environment, 39(1), 17-25, doi:10.1016/j.atmosenv.2004.09.054, 2005.

Yanowitz, J., McCormick, R. L. and Graboski, M. S.: In-Use Emissions from Heavy-Duty Diesel Vehicles, Environmental Science \& Technology, 34(5), 729-740, doi:10.1021/es990903w, 2000.

10 Zhang, Q., Klimont, Z., Streets, D., Huo, H. and He, K.: An anthropogenic PM emission model for China and emission inventory for the year 2001, Progress in Natural Science, 16(2), 223-231 (in Chinese), 2006.

Zhang, Q., Streets, D. G., Carmichael, G. R., He, K. B., Huo, H., Kannari, A., Klimont, Z., Park, I. S., Reddy, S., Fu, J. S., Chen, D., Duan, L., Lei, Y., Wang, L. T. and Yao, Z. L.: Asian emissions in 2006 for the NASA INTEX-B mission, Atmos. Chem. Phys., 9, 5131-5153, 2009.

15 Zhang, Y., Schauer, J. J., Zhang, Y., Zeng, L., Wei, Y., Liu, Y. and Shao, M.: Characteristics of Particulate Carbon Emissions from Real-World Chinese Coal Combustion, Environ. Sci. Technol., 42(14), 5068-5073, doi:10.1021/es7022576, 2008.

Zhang, Z.: Energy efficiency and environmental pollution of brickmaking in China, Energy, 22(1), 33-42, 1997.

Zhi, G., Peng, C., Chen, Y., Liu, D., Sheng, G. and Fu, J.: Deployment of Coal Briquettes and Improved Stoves: Possibly an 20 Option for both Environment and Climate, Environ. Sci. Technol., 43(15), 5586-5591, doi:10.1021/es802955d, 2009.

Zhu, D., Nussbaum, N. J., Kuhns, H. D., Chang, M. C. O., Sodeman, D., Uppapalli, S., Moosmüller, H., Chow, J. C. and Watson, J. G.: In-Plume Emission Test Stand 2: Emission Factors for 10- to 100-kW U.S. Military Generators, Journal of the Air \& Waste Management Association, 59(12), 1446-1457, doi:10.3155/1047-3289.59.12.1446, 2009. 